\title{
Supporting information for: PbS Nanocrystals made using Excess Lead Chloride have a Halide-Perovskite-like Surface
}

Philippe B. Green ${ }^{1}$, Francisco Y. Villanueva ${ }^{1}$, Karl Z. Demmans ${ }^{1}$, Christian J. Imperiale ${ }^{1}$, Minhal Hasham $^{1}$, Ehsan Nikbin ${ }^{2}$, Jane Y. Howe ${ }^{2}$, Darcy C. Burns ${ }^{1}$, and Mark W.B. Wilson ${ }^{1 *}$

1) Department of Chemistry, University of Toronto, Toronto, Ontario M5S 3H6, Canada

2) Department of Materials Science and Engineering, University of Toronto, Toronto, Ontario, M5S 3E4, Canada

*mark.w.b.wilson@utoronto.ca

\section{Contents}

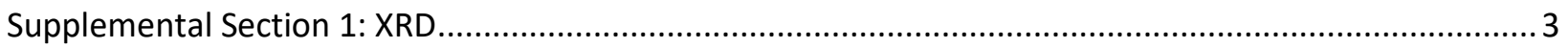

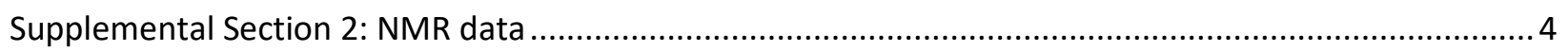

Supplemental Section 3: Additional NC Characterization .................................................................. 19

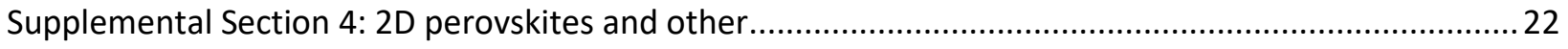

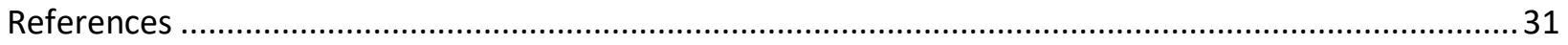


Case 1: Exchange between oleylammonium-halides and oleylammonium-oleates

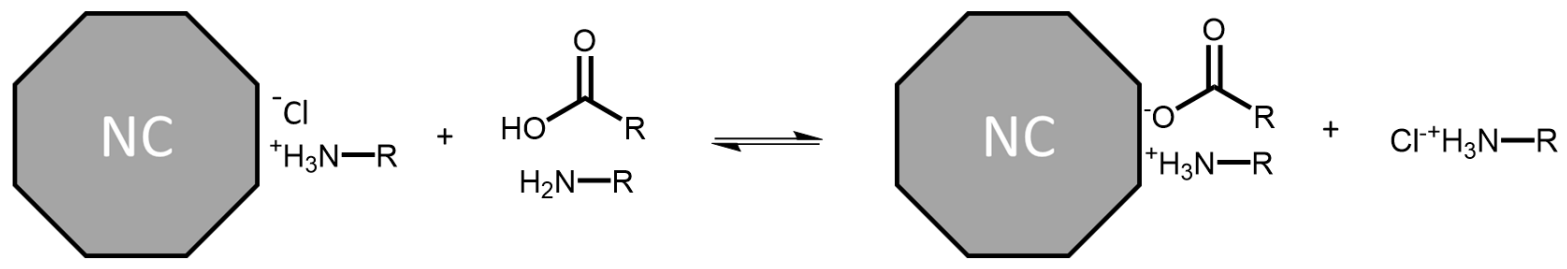

Case 2: Displacement of an oleylammonium-halide by a carboxylic acid

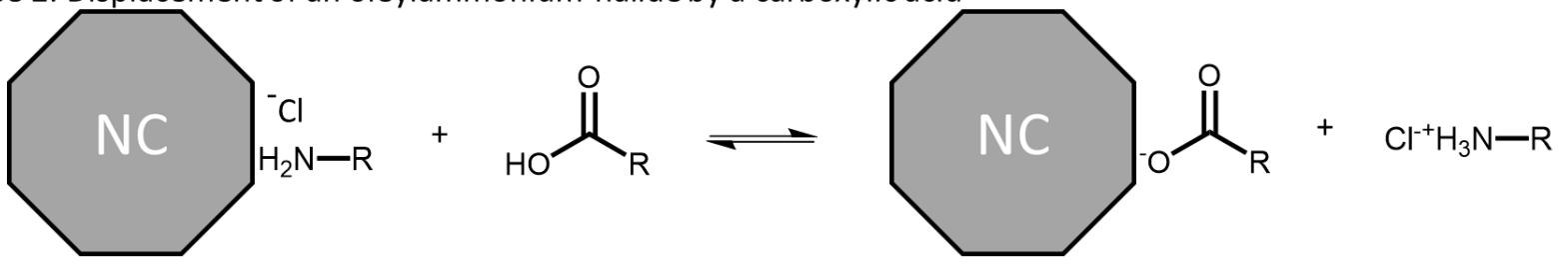

Case 3: Dissociative absorption of carboxylic acids on the surface

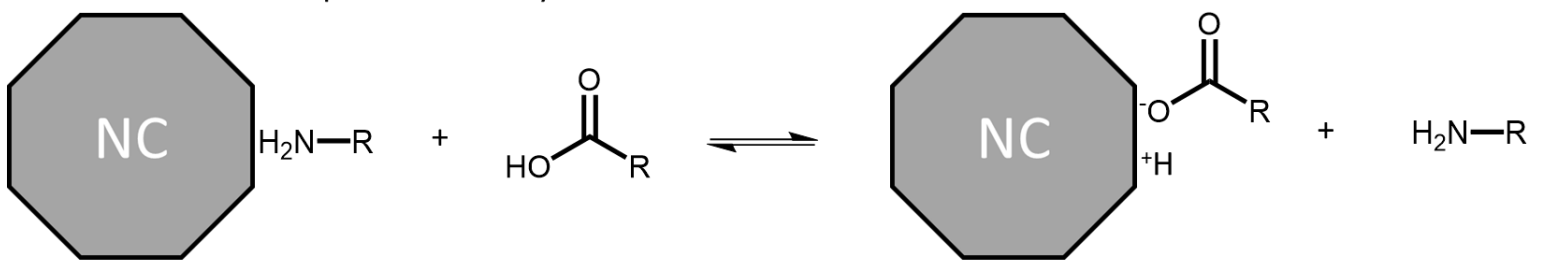

Case 4: L-type binding of carboxylic acids

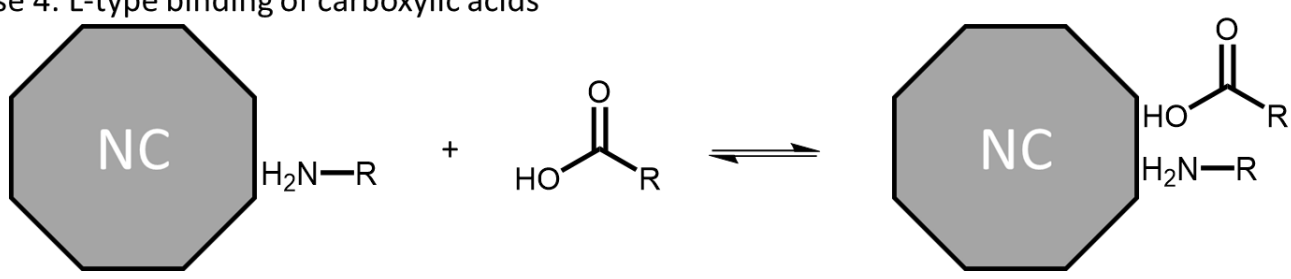

Scheme S1: Schematic representation of the four proposed exchanges that could occur at the surface of nanocrystal with an initial oleylamine/halide passivation. We conclude that Case 1 is the dominant exchange observed. 


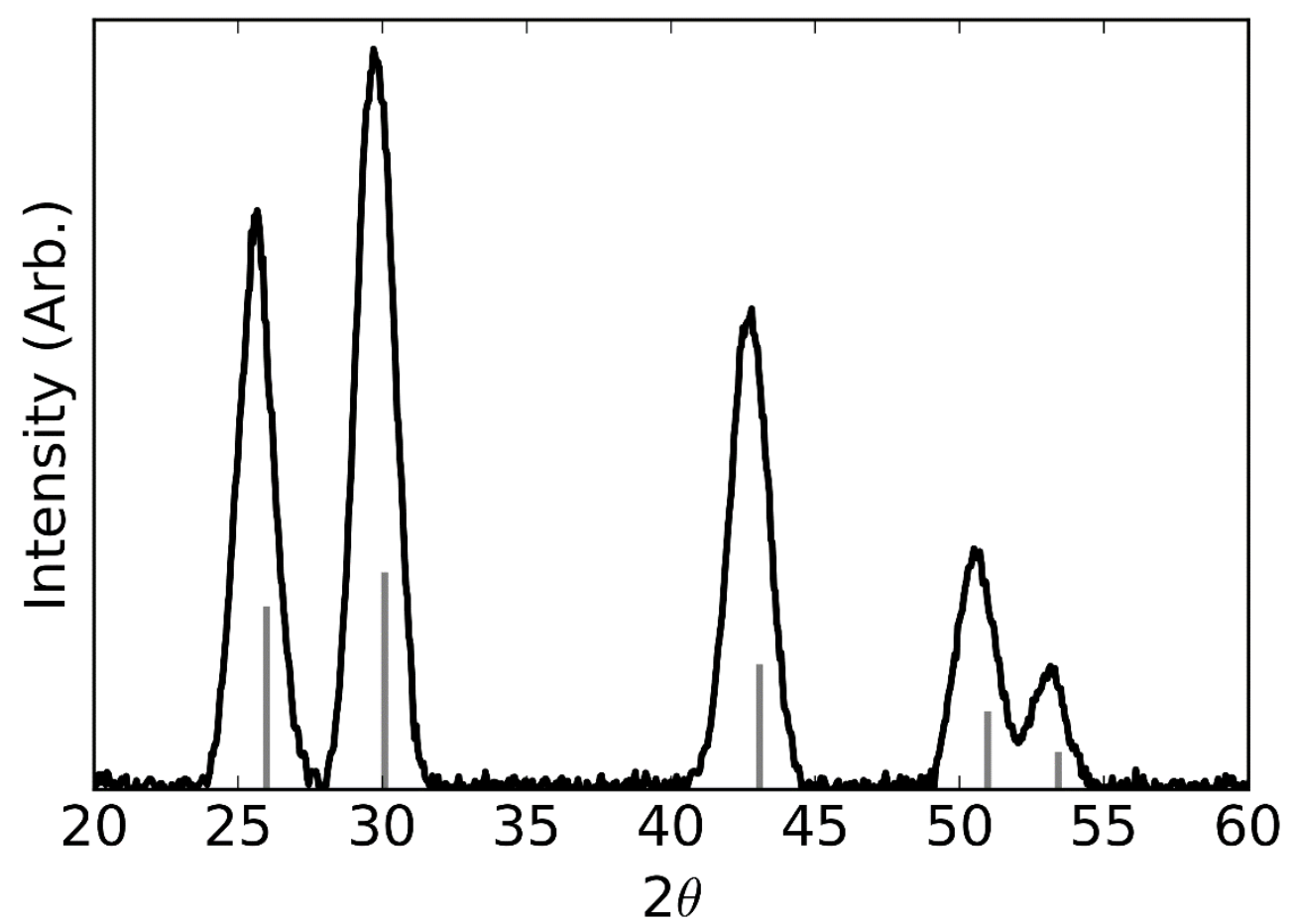

Figure S1: XRD pattern for PbS-eCl NCs purified through our acetone washes (See methods). The pattern matches that expected for PbS (grey lines, ICDD PDF number 00-005-0592). We observe no signature of crystalline $\mathrm{PbCl}_{2}$. This confirms that our purification methods yields a high purity sample. ${ }^{1}$ 

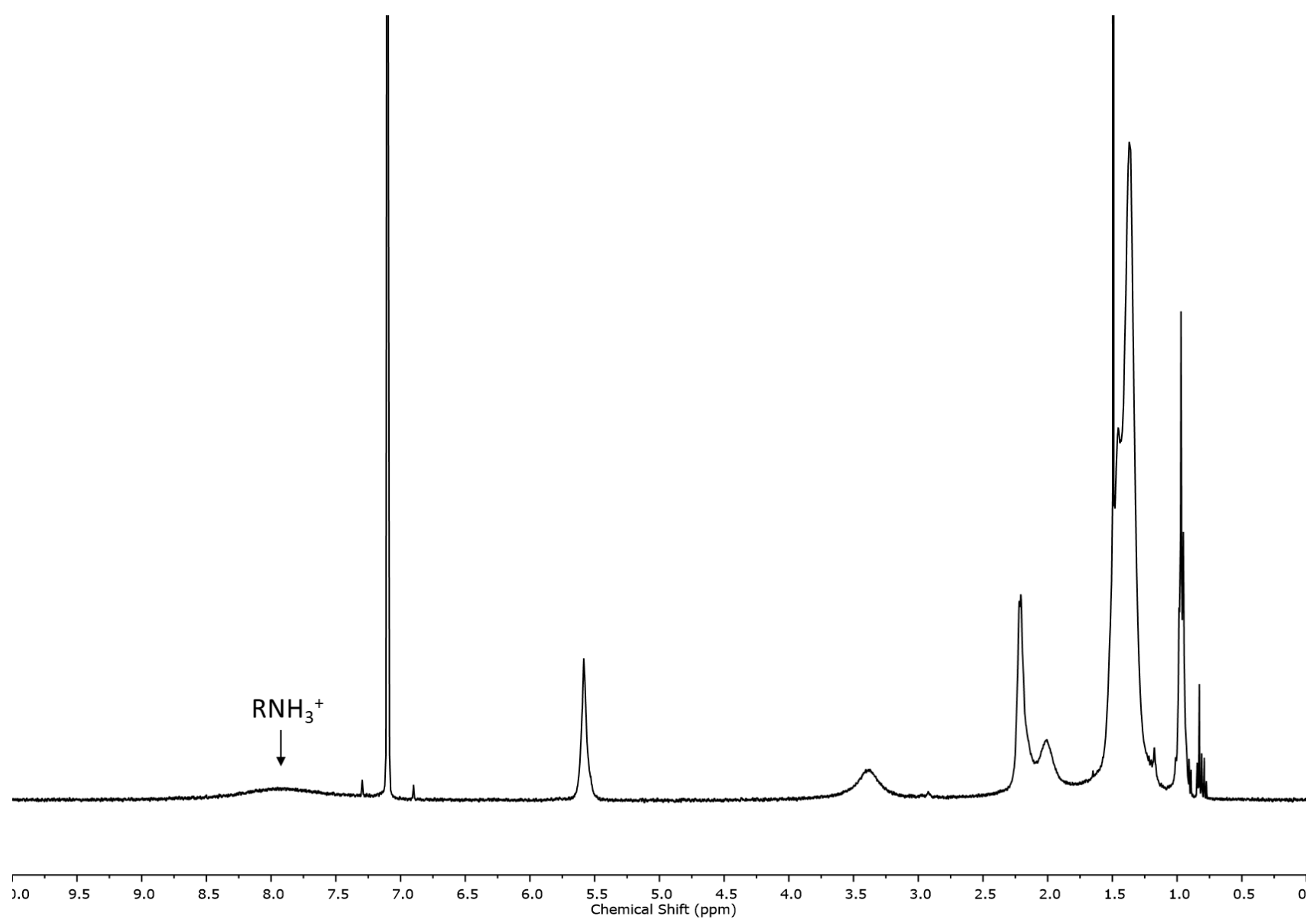

Figure S2: Complete ${ }^{1} \mathrm{H}$ NMR of a native PbS-eCl NCs ensemble also shown in figure $1 \mathrm{C}$ and $2 \mathrm{~A}$. Of particular importance is the oleylammonium $\left(\mathrm{RNH}_{3}{ }^{+}\right)$resonance. The presence of this resonance suggests that PbS-eCl NCs are passivated by oleylammonium instead of L-type OLA. 


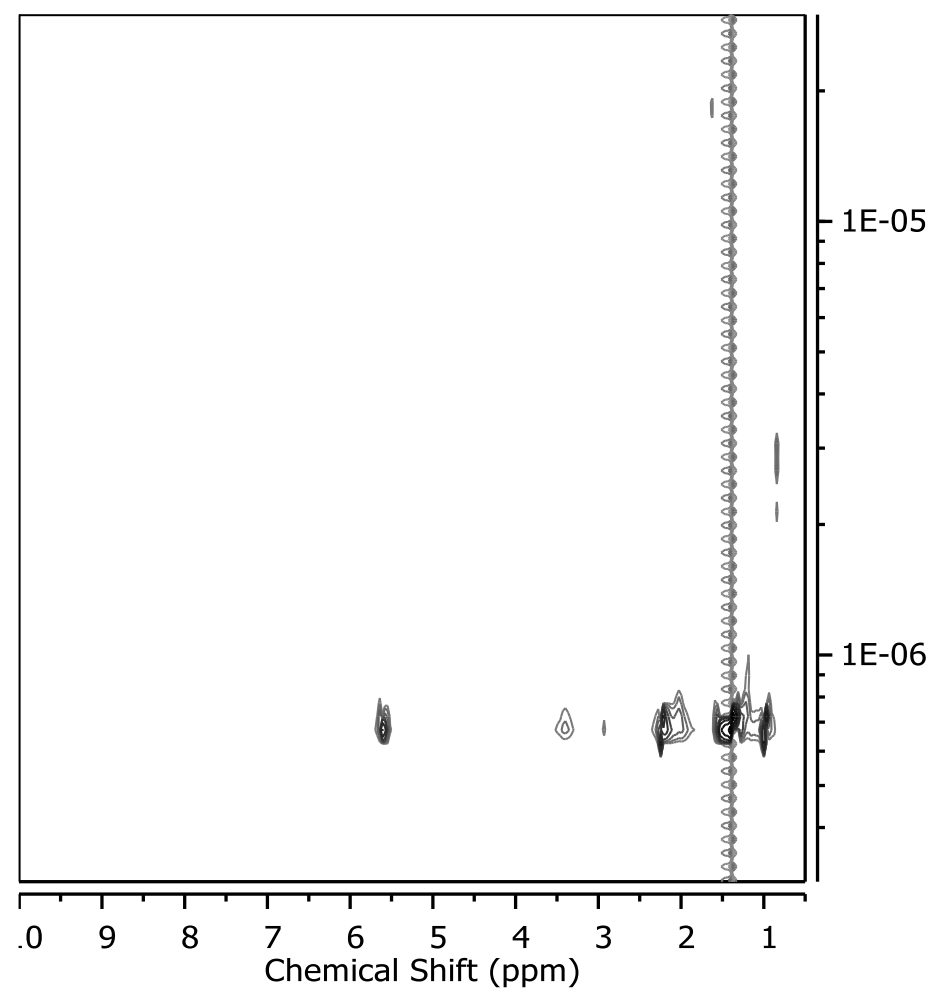

Figure S3: DOSY of native PbS-eCl NCs, the complete corresponding ${ }^{1} \mathrm{H}$ NMR is displayed in figure S2 and excerpts are shown in the main text in figure 1. 


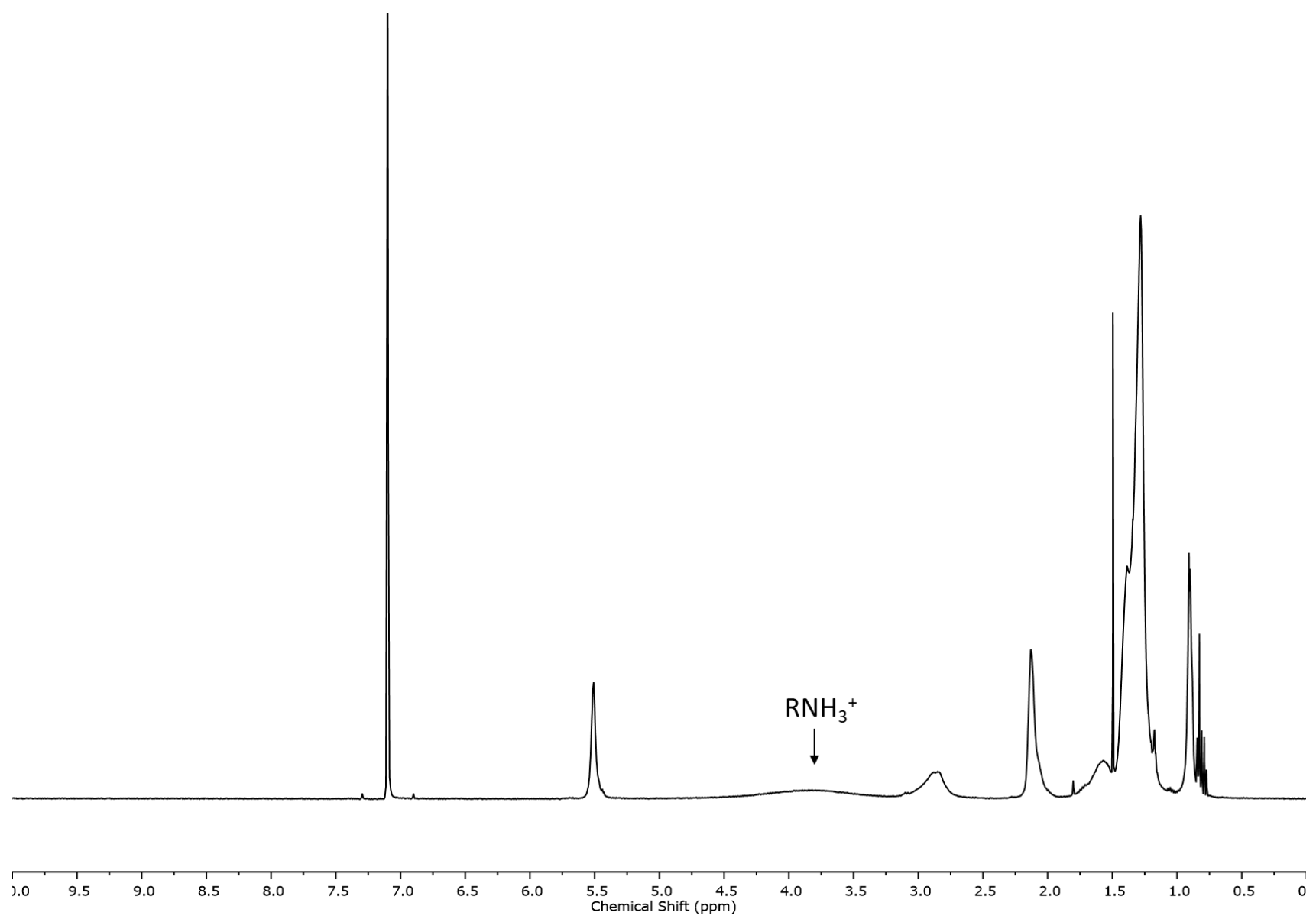

Figure S4: Complete ${ }^{1} \mathrm{H}$ NMR of a native PbS-eCl NC ensemble with added OLA (50umol/mL) also shown in figure $2 \mathrm{~A}$. The oleylammonium resonance is drastically shifted upfield suggesting a dynamic equilibrium between ammonium and amine species. Given that we observe that ligands are preferentially bound in purified solutions prior to the addition of free OLA, this suggests that amines are also in equilibrium with the surface. 


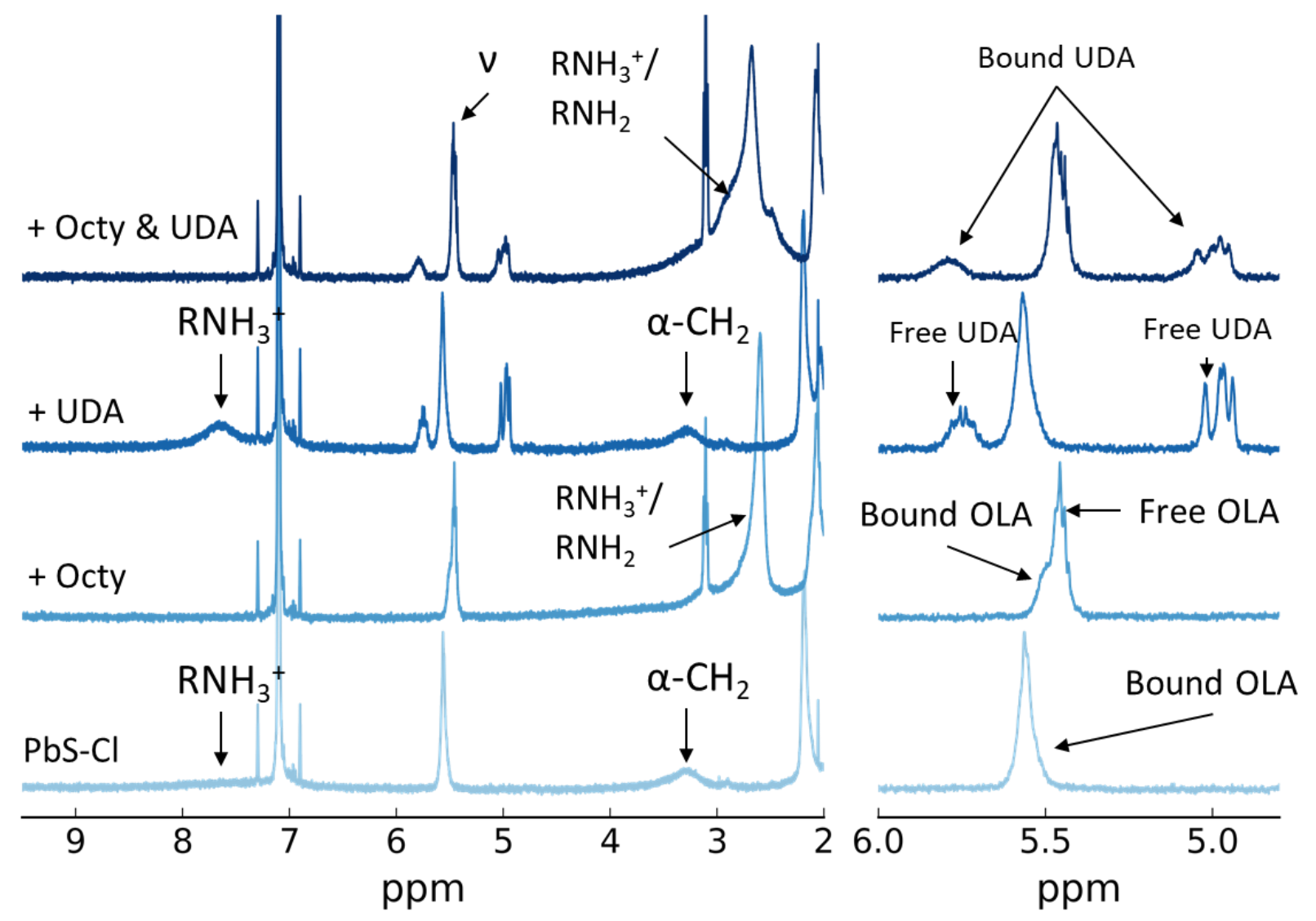

Figure S5: Similar exchanges as presented in the main manuscript but performed by adding octylamine (Octy) instead of OLA. To the left is the portion of the spectrum showing the ammonium peaks, while to the right only the alkene resonances are shown. The bottom spectrum is of the native PbS-eCl NCs. The addition of Octy shifts the ammonium peak upfield, consistent with a new equilibrium between bound ammonium and free amines. All other resonances are also shifted upfield, indicating that they are now in equilibrium between free and bound states. In contrast, the addition of UDA does not shift any of the OLA resonances. However, the ammonium peaks is narrower and we ascribe this to physiosorbed UDA. This is consistent with UDA remaining free in solution. When both Octy and UDA are co-added, the alkene resonances of UDA are seen to broaden indicative that UDA is now bound. This set of observations is parallel to the effect of OLA discussed in the main text, and corroborates our picture where the native passivation of $\mathrm{PbS}-\mathrm{eCl} \mathrm{NCs}$ involves ammonium and chloride, which can be jointly exchanged for ammonium-carboxylates, or undergo ammonium-for-amine exchange promoted by proton transfer at the surface. 


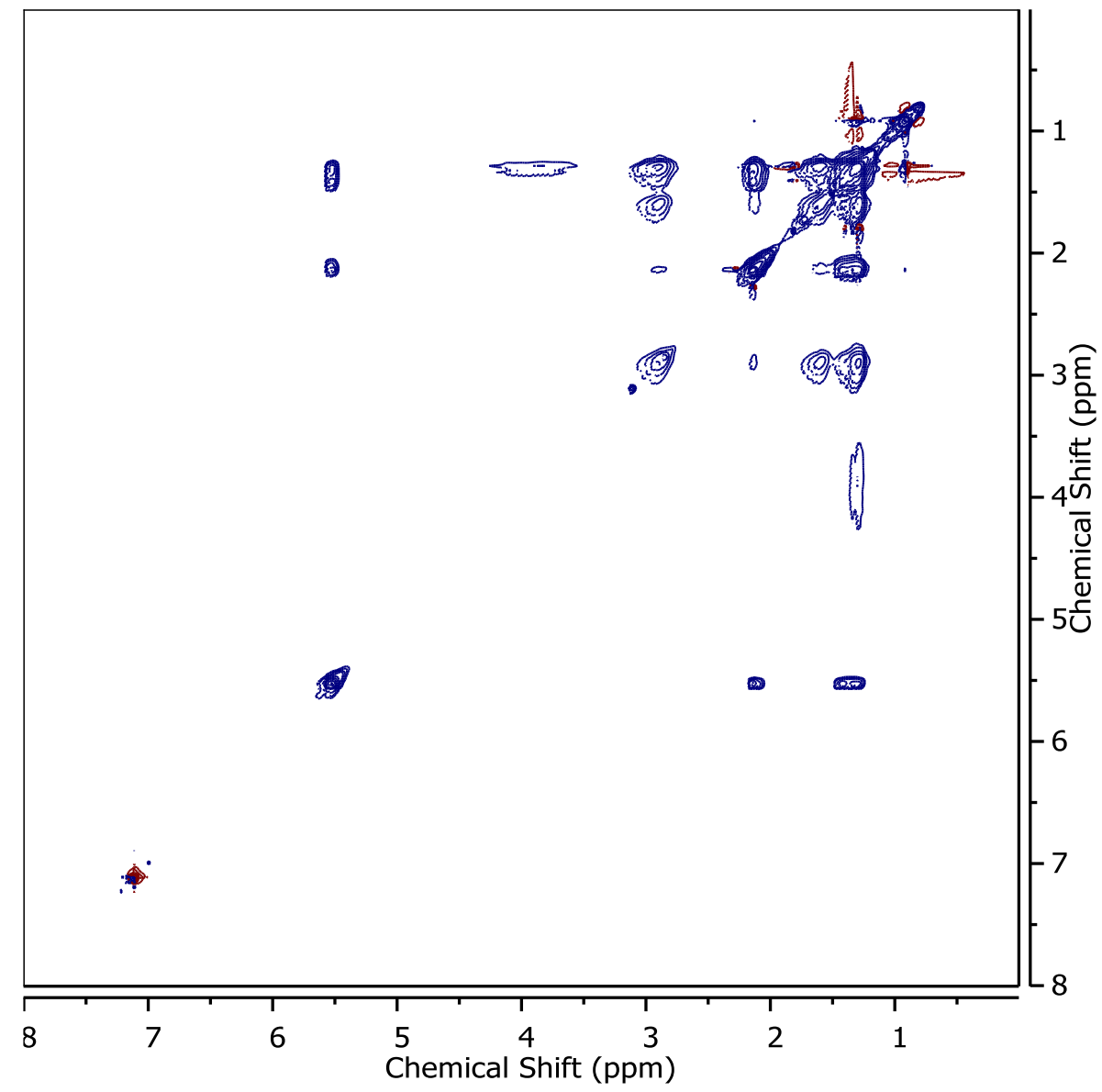

Figure S6: 2D NOESY of PbS-eCl NCs with added OLA, the complete corresponding ${ }^{1} \mathrm{H}$ NMR is displayed in figure $\mathrm{S} 4$ and excerpts are shown in the main text in figure 2. Negative cross-peaks are observed between all OLA resonances. This shows that excess free OLA is in equilibrium with bound oleylammonium on the surface of PbS-eCl NCs. 


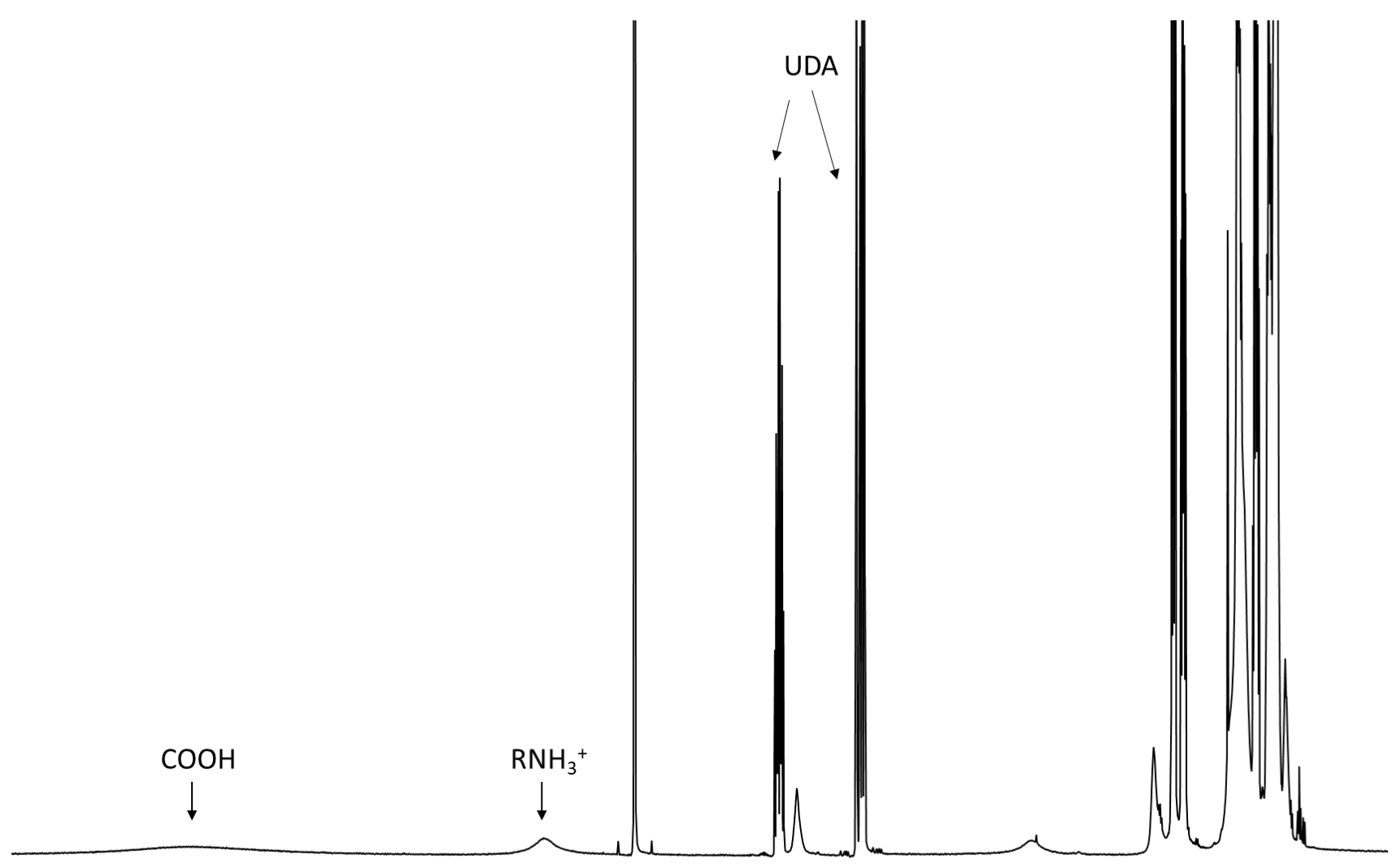

$\begin{array}{llllllllllllllllllllllllllllllllll}12.5 & 12.0 & 11.5 & 11.0 & 10.5 & 10.0 & 9.5 & 9.0 & 8.5 & 8.0 & 7.5 & 7.0 & 6.5 & 6.0 & 5.5 & 5.0 & 4.5 & 4.0 & 3.5 & 3.0 & 2.5 & 2.0 & 1.5 & 1.0 & 0.5 & 0\end{array}$

Figure S7: Complete ${ }^{1} \mathrm{H}$ NMR of a native PbS-eCl NC ensemble with added UDA ( $\left.2 \mathrm{mmol} / \mathrm{mL}\right)$ as shown in figure $2 \mathrm{~A}$ and $3 \mathrm{~A}$. The UDA resonances remain narrow suggesting that UDA remains free in solution. The ammonium resonance is scarcely affected (c.f. Figure S2), though the very slight downfield shift, is consistent with some amount of proton transfer between the bound oleylammonium and a small population of physisorbed UDA. 

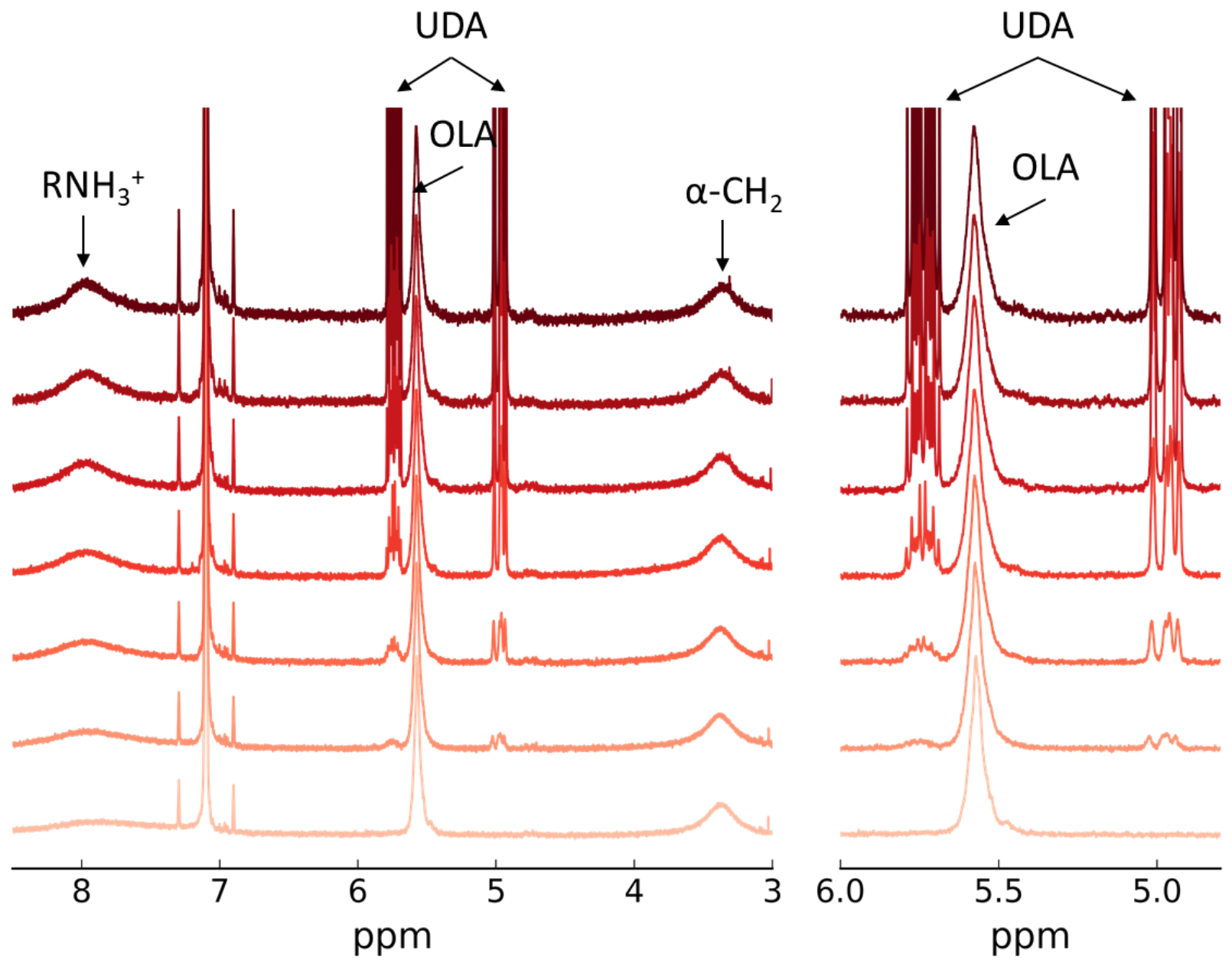

Figure S8: Titration of an ensemble of PbS-eCl NCs with UDA. As can be seen on the right, UDA does not result in upfield shifting of the OLA resonances during the titration. The UDA resonances remain narrow while that of OLA remains broad. This is consistent with UDA (alone) being unable to bind the surface and leaving the native surface unaffected. Again we note the ammonium peak at around $8 \mathrm{ppm}$ slightly shifts downfield and narrows with increasing UDA. This is consistent with proton exchanges from physisorbed UDA and bound oleylammonium but without binding of UDA. 


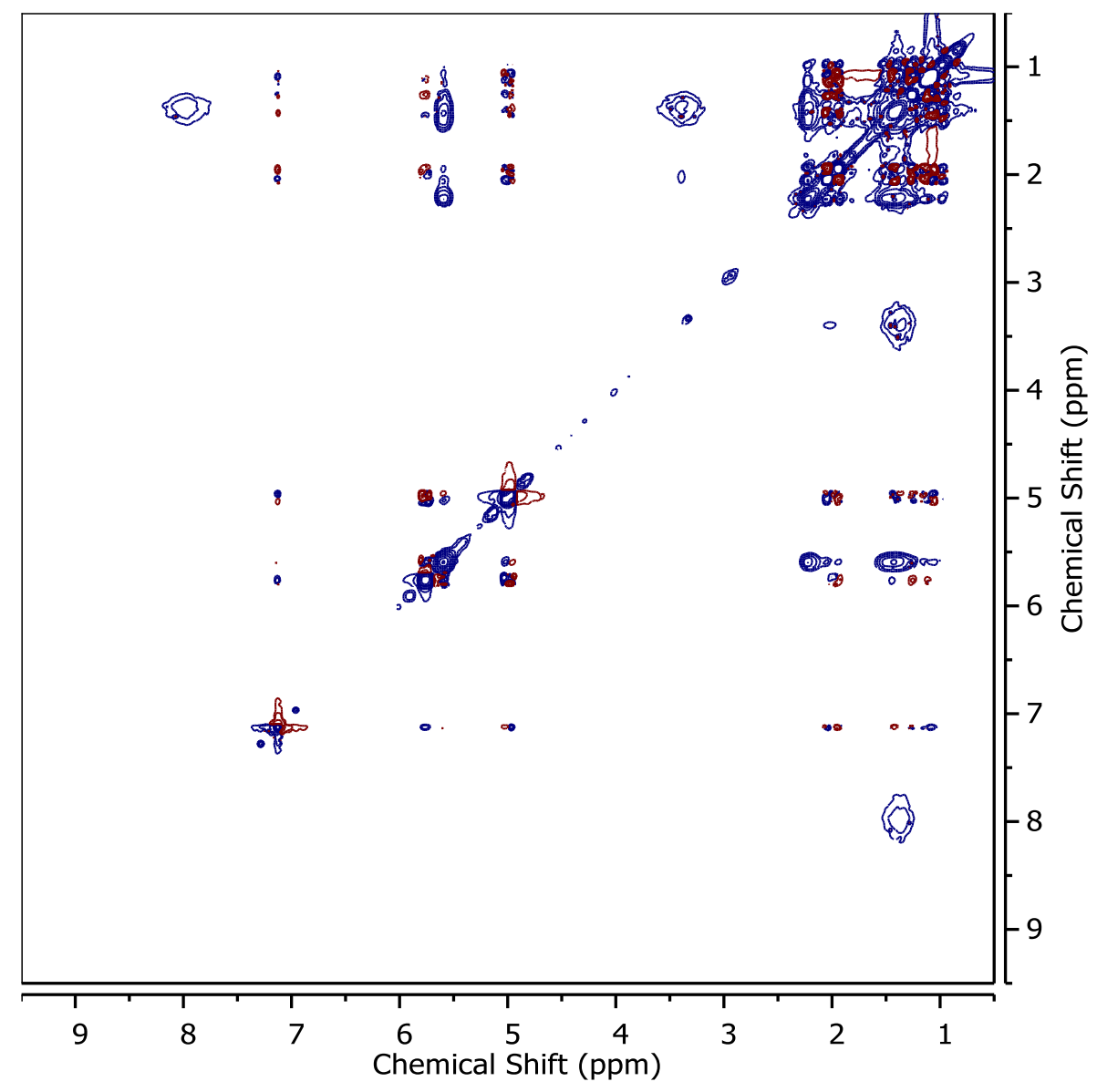

Figure S9: 2D NOESY of PbS-eCl NCs with added UDA, the complete corresponding ${ }^{1} \mathrm{H}$ NMR is displayed in figure $\mathrm{S} 7$ and excerpts are shown in the main text in figure 2. Negative crosspeaks are observed for all OLA resonances. This indicates that the OLA still interacts with the NCs surface. UDA resonances $(5.75,4.97$, $2.02,1.93,1.40,1.21 \mathrm{ppm}$ ) display positive crosspeaks (red) showing that it is free in solution. This is particularly visible in the alkyl region (0.5-2.2 ppm) were positive peaks are now observed (in contrast to the behaviour of OLA, see Figure S4, or OLA+UDA, Figure S10). 


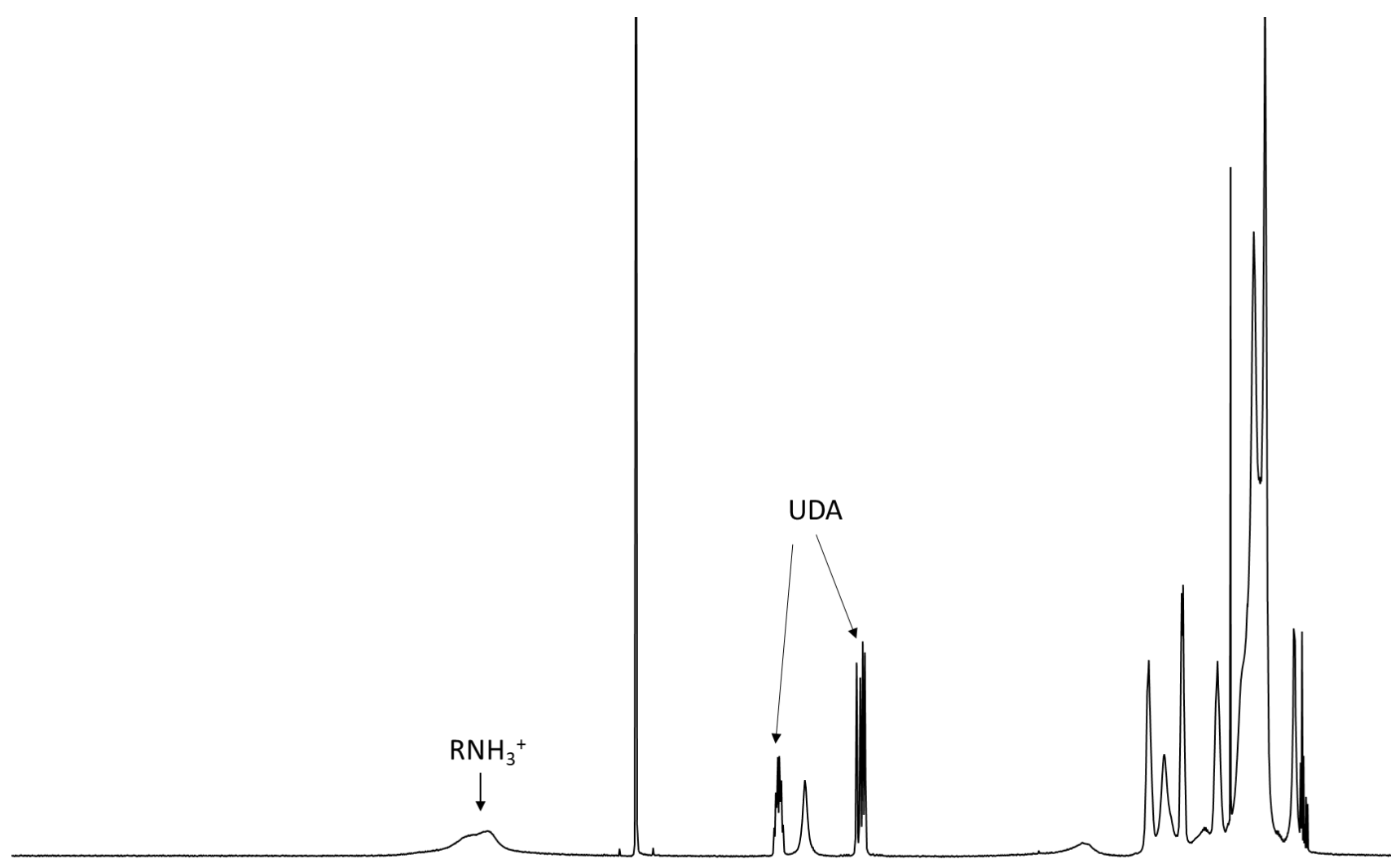

$\begin{array}{lllllllllllllllllllllllllll}12.5 & 12.0 & 11.5 & 11.0 & 10.5 & 10.0 & 9.5 & 9.0 & 8.5 & 8.0 & 7.5 & 7.0 & 6.5 & 6.0 & 5.5 & 5.0 & 4.5 & 4.0 & 3.5 & 3.0 & 2.5 & 2.0 & 1.5 & 1.0 & 0.5 & 0\end{array}$

Figure S10: Complete ${ }^{1} \mathrm{H}$ NMR of a native PbS-eCl NCs ensemble with added OLA+UDA as shown in figure 3A. All the UDA resonances are broadened, suggesting that UDA can penetrate the ligand shell and is now in equilibrium between its free and bound state. The ammonium peak is no longer represented by a single resonance. Instead, a multi-peaked feature is observed that is well fitted with three distinct resonances (See figure S20). This is to be expected as the initially bound oleylammonium can be displaced through the formation of oleylammonium-carboxylate or oleylammonium-chloride. 


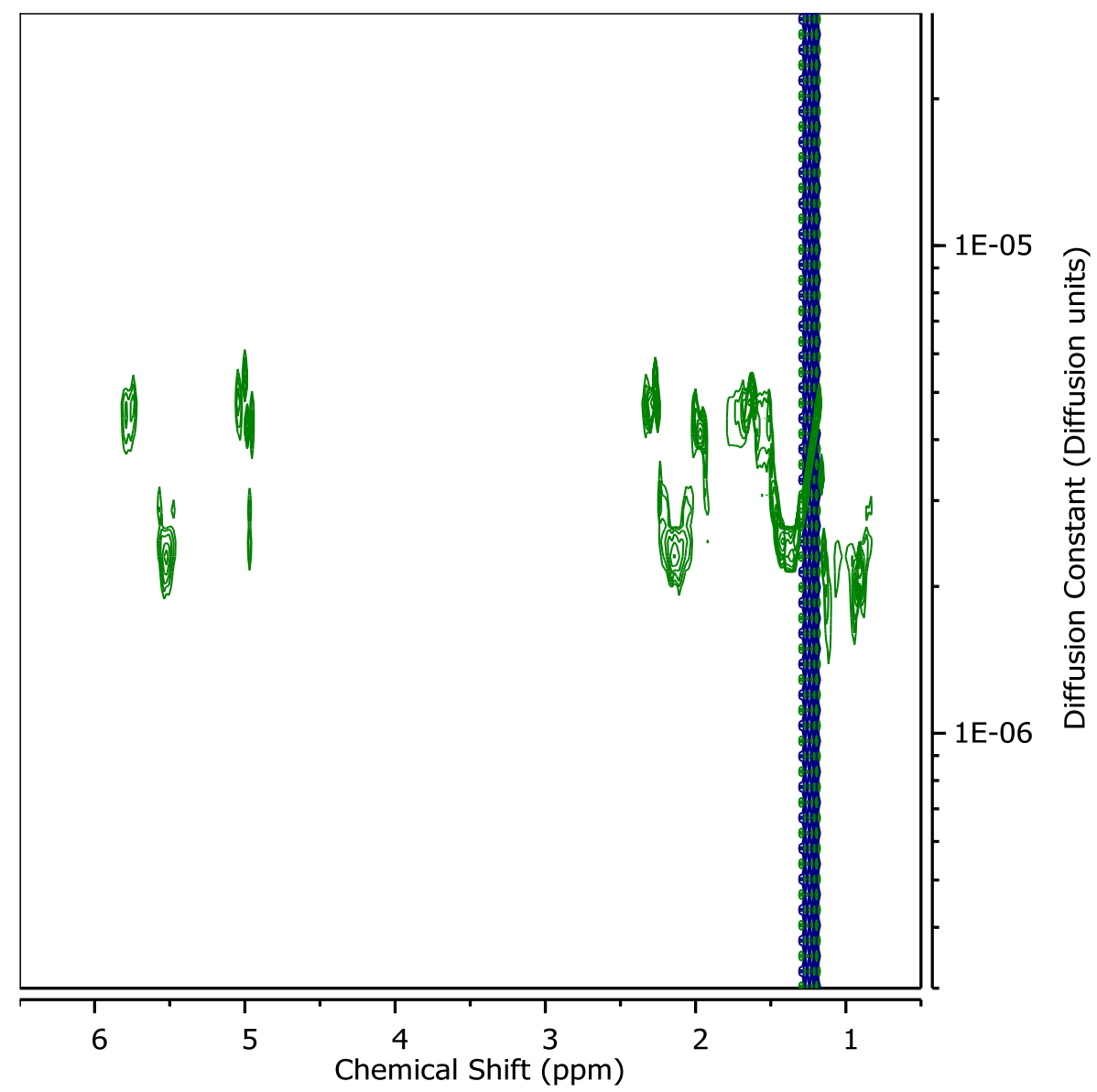

Figure S11: DOSY of PbS-eCl NCs with added OLA+UDA, the complete corresponding ${ }^{1} \mathrm{H}$ NMR is displayed in figure $\mathrm{S} 10$ and excerpts are shown in the main text in figure 3. 


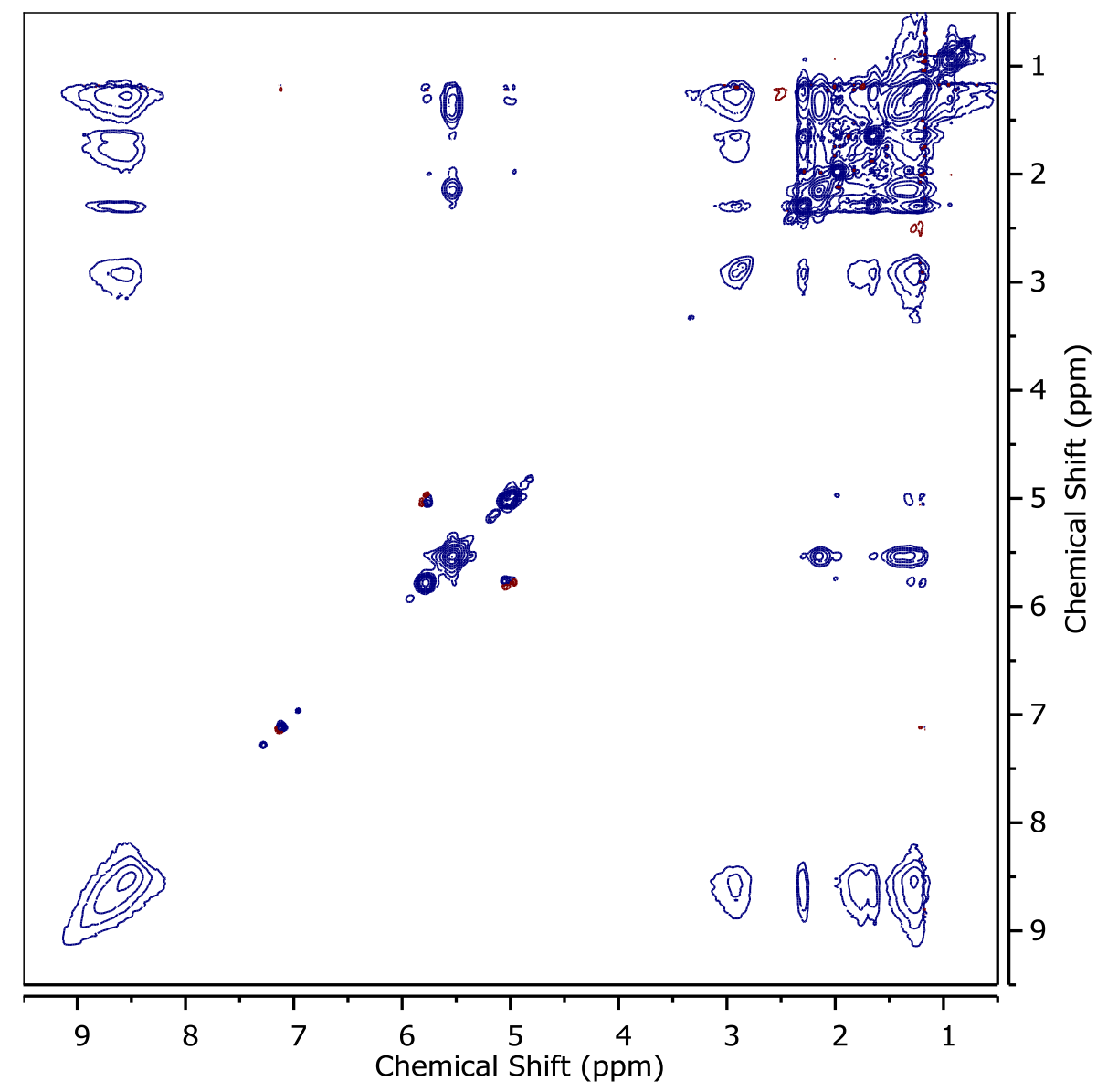

Figure S12: 2D NOESY of PbS-eCl NCs with added OLA+UDA, the complete, corresponding ${ }^{1} \mathrm{H}$ NMR is displayed in figure S10 and excerpts are shown in the main text in figure 3. Negative crosspeaks are observed for all OLA and UDA resonances. This indicates that the OLA and UDA interacts with the NCs surface and that all ligand species are in equilibrium with the surface of PbS-eCl NCs. It is useful to contrast this spectrum with the 2D NOESY acquired for PbS-eCl NCs with the addition of only UDA (Figure S7). In the UDA-only experiments, positive crosspeaks could be observed for all the UDA resonances while negative ones were observed for OLA. In this experiment no positive peaks can be observed. 

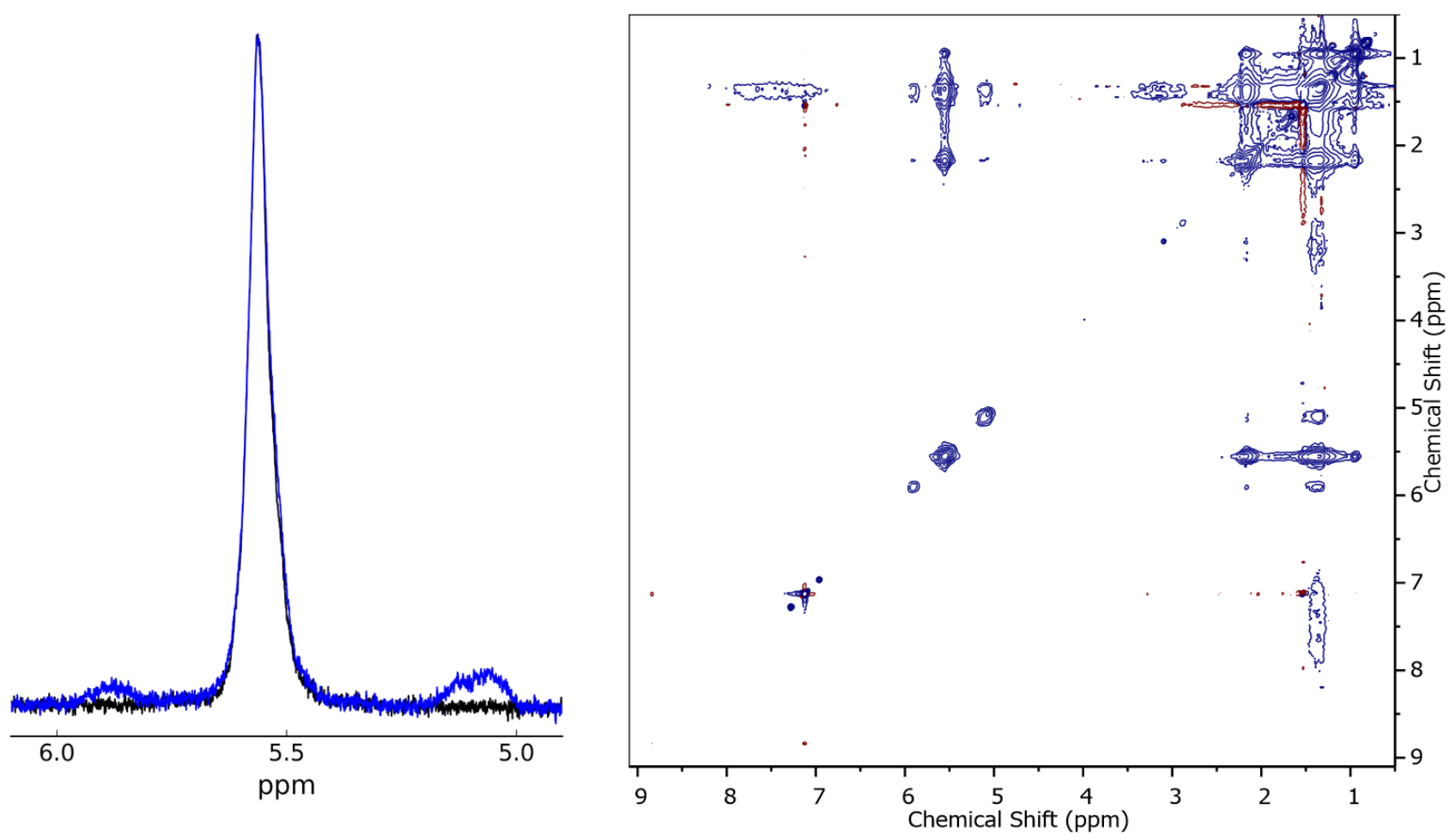

Figure S13: After purification, residual bound UDA coverage is observed only if OLA+UDA are introduced simultaneously. Only low UDA coverages could be obtained in these experiments, as higher amounts rendered the NCs insoluble. (Left) The alkene region of purified NCs that where exposed to UDA only (black) and those exposed to OLA+UDA (blue). Following this purification, no signatures of UDA (resonances at 5.1 and $5.8 \mathrm{ppm}$ ) are observed. By contrast, if OLA+UDA are added together, UDA remains present even following purification, confirming that it is bound to the NC surface. The 2D NOESY of the sample previously exposed to OLA+UDA, confirms that UDA is bound through the presence of clear negative cross peaks. 


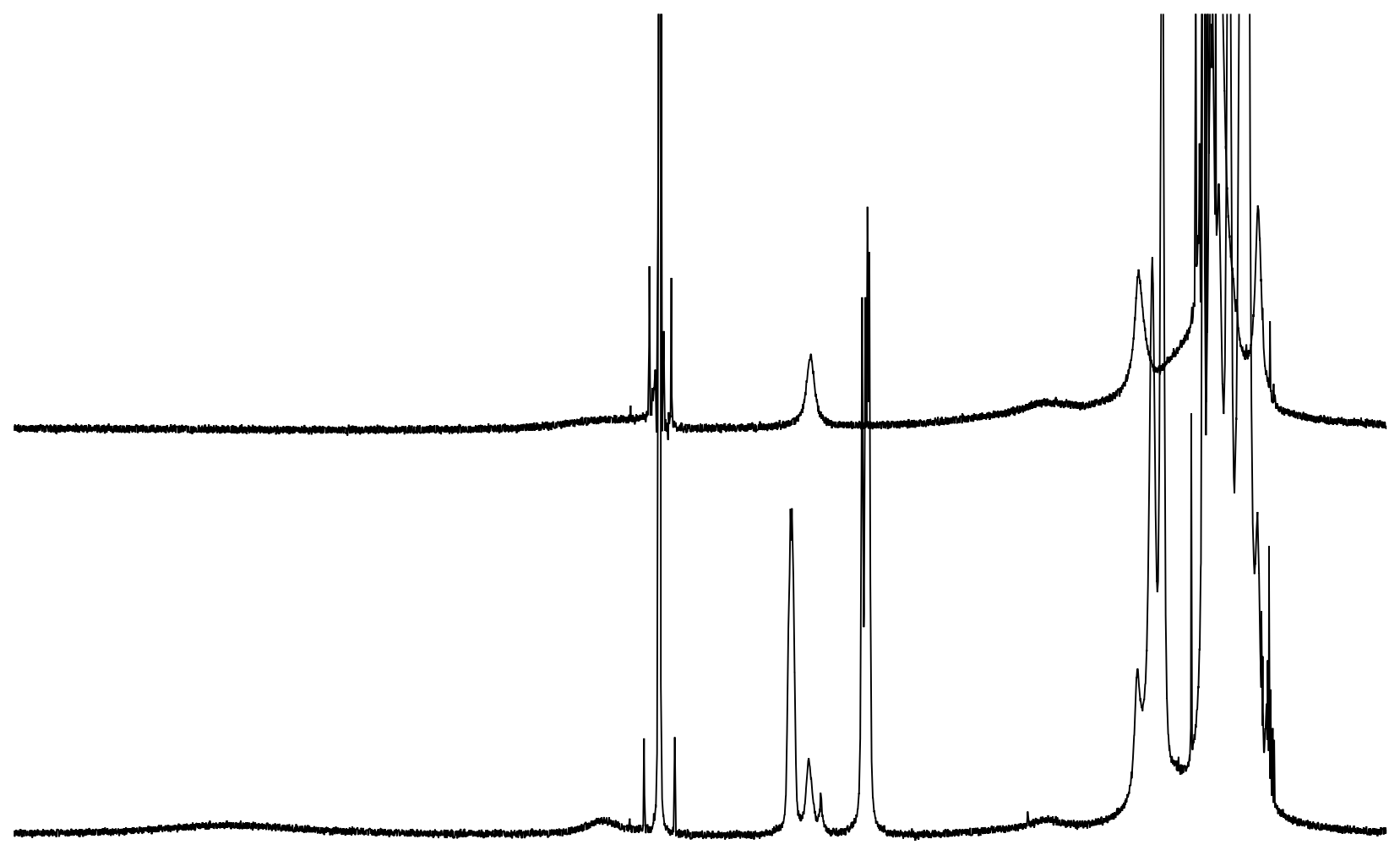

$\begin{array}{llllllllllllllllllllllllllllll}13.5 & 13.0 & 12.5 & 12.0 & 11.5 & 11.0 & 10.5 & 10.0 & 9.5 & 9.0 & 8.5 & 8.0 & 7.5 & 7.0 & 6.5 & 6.0 & 5.5 & 5.0 & 4.5 & 4.0 & 3.5 & 3.0 & 2.5 & 2.0 & 1.5 & 1.0 & 0.5 & 0.0\end{array}$

Figure S14: Complete ${ }^{1} \mathrm{H}$ NMR of a PbS-eCl NC ensemble (Top) exposed to OLA+OA and then purified and then (bottom) with added UDA $(2 \mathrm{mmol} / \mathrm{mL})$. The excerpt of the alkene region is shown in Figure 4. 


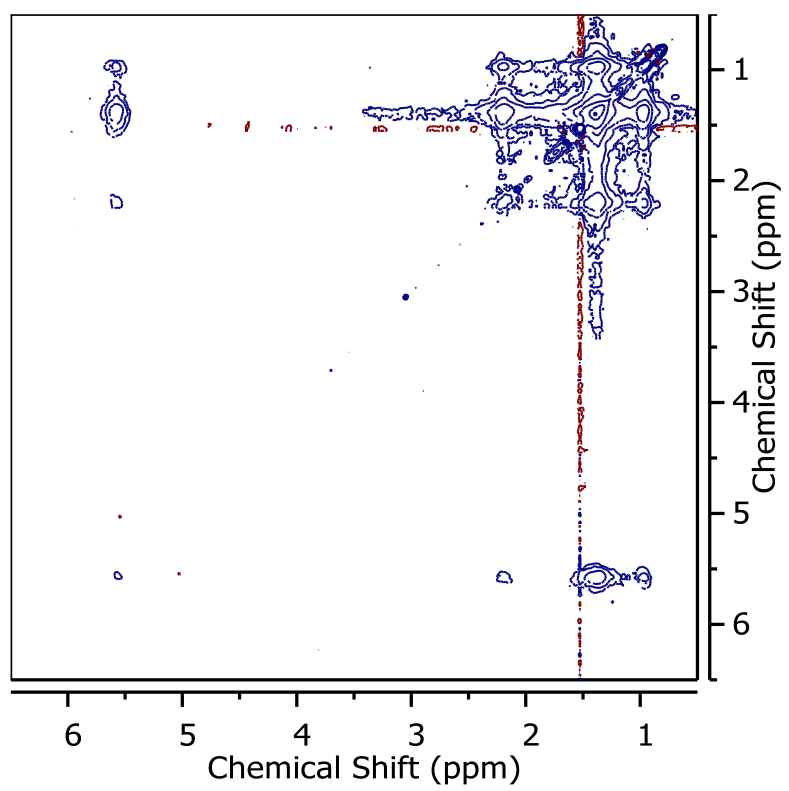

Figure S15: 2D NOESY of purified PbS-eCl NCs with added OLA+OA, the complete corresponding ${ }^{1} \mathrm{H}$ NMR is displayed in figure S14 and an excerpt is shown in the main text in figure 4. Negative crosspeaks are observed for all OLA and OA resonances. This indicates that the OLA and OA are interacting with the surface of the NCs. 


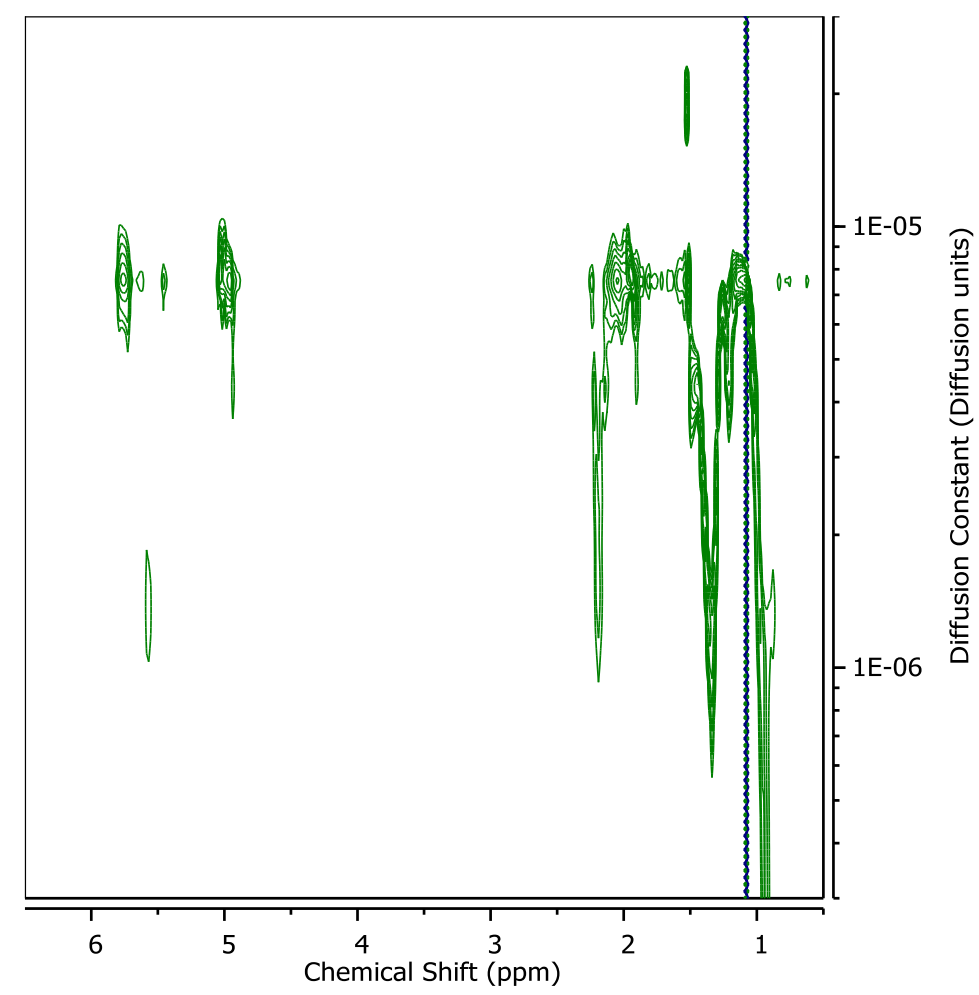

Figure S16: DOSY of purified PbS-eCl NCs with added OLA+OA and then subjected to UDA, the complete, corresponding ${ }^{1} \mathrm{H}$ NMR is displayed in figure $\mathrm{S} 14$ and an excerpt is shown in the main text in figure 4 . These data show that the carboxylates are in equilibrium between free and bound, but are predominantly free on average. In contrast, the OLA is predominantly bound.

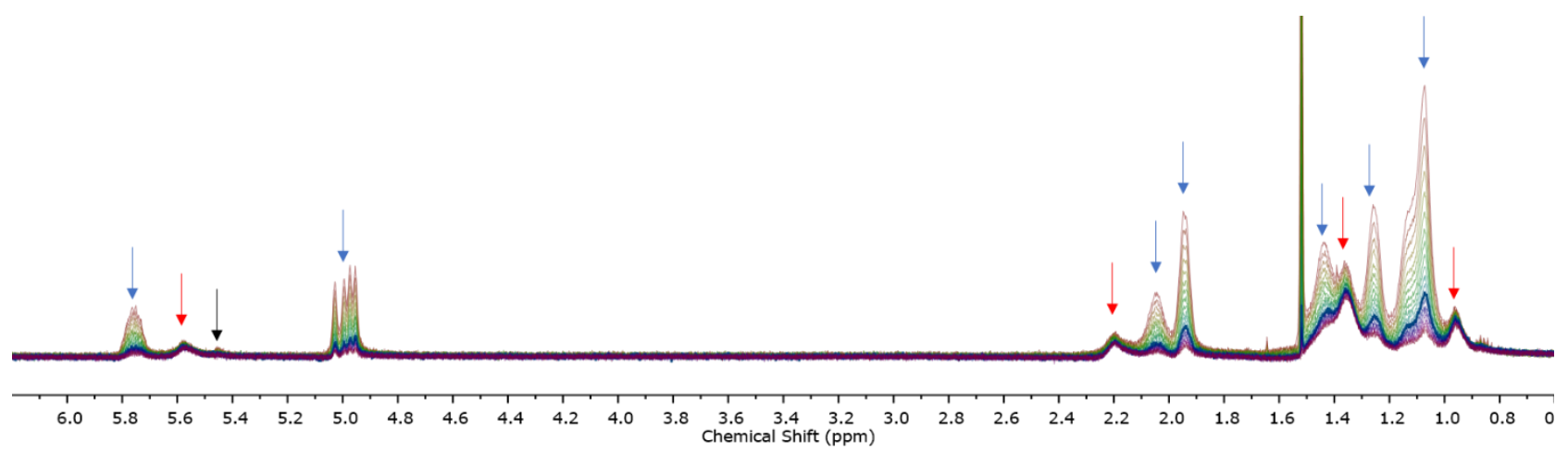

Figure S17: Raw ${ }^{1} \mathrm{H}$ NMR data of purified PbS-eCl NCs with added OLA+OA and then subjected to UDA, which was used to perform DOSY as shown in Figure S16. The distinct field-gradient dependences of UDA peaks (blue arrows), compared to OLA peaks (red arrows) shows that the former is predominantly free, and the latter is predominantly bound. The only observable peak uniquely corresponding to OA is identified with a black arrow. Upon close inspection, we observe that it has a similarly strong field-gradient dependence to the UDA, suggesting that it is also predominantly free. 


\section{Supplemental Section 3: Additional NC Characterization}
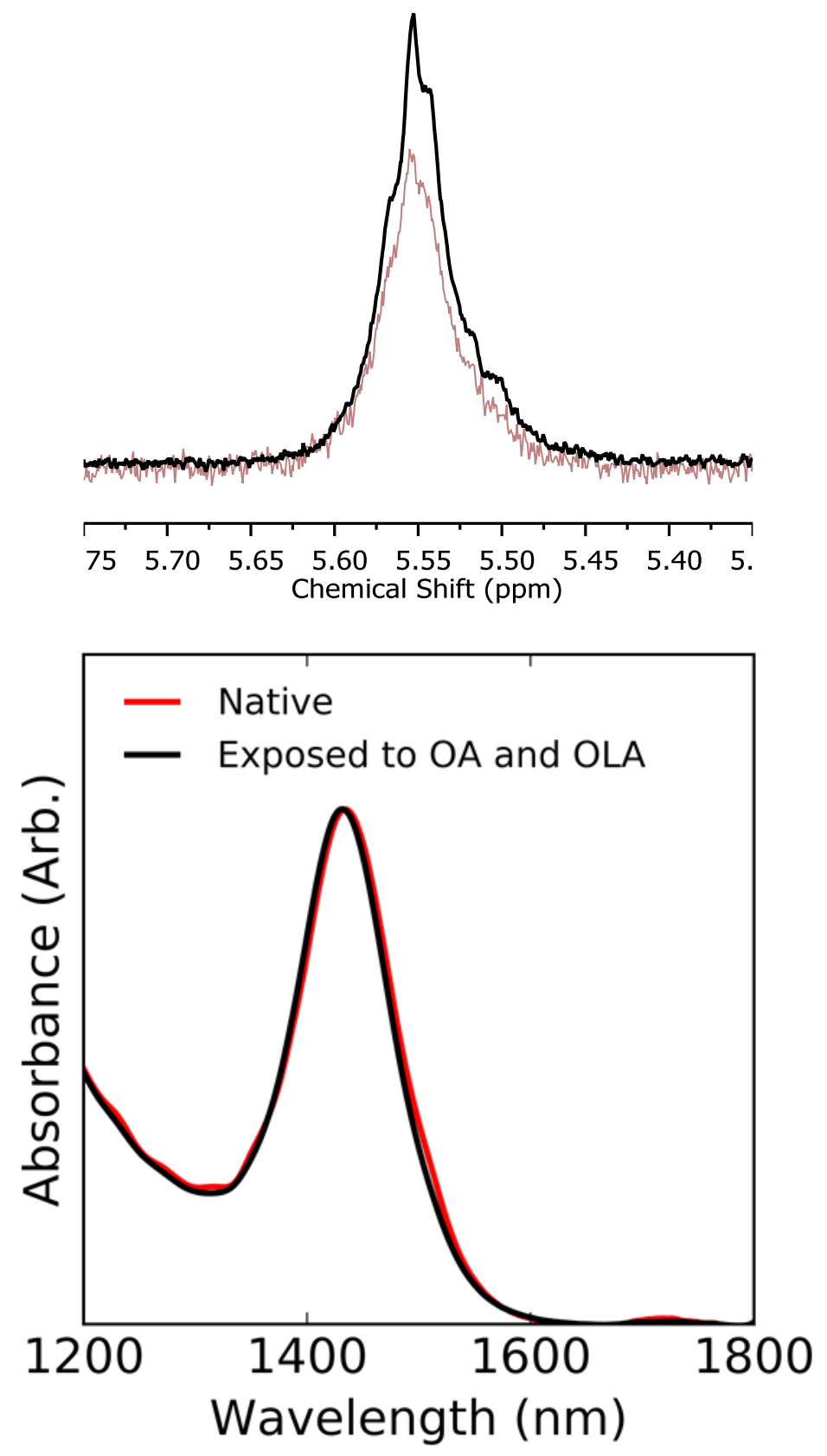

Figure S18: Top: ${ }^{1} \mathrm{H}$ NMR spectrum of the alkene peak of PbS-eCl NCs as obtained (red) or exposed and purified to OLA+OA. A 1.7-fold increase in aliphatic ligand coverage is observed. These spectra were normalized via the ferrocene standard and calculated concentration via optical absorption at 400nm. Bottom: Optical absorption spectrum of PbS-eCl NCs. Native NCs are shown in red while NCs exposed to OLA+OA in black. No significant difference is observed. 

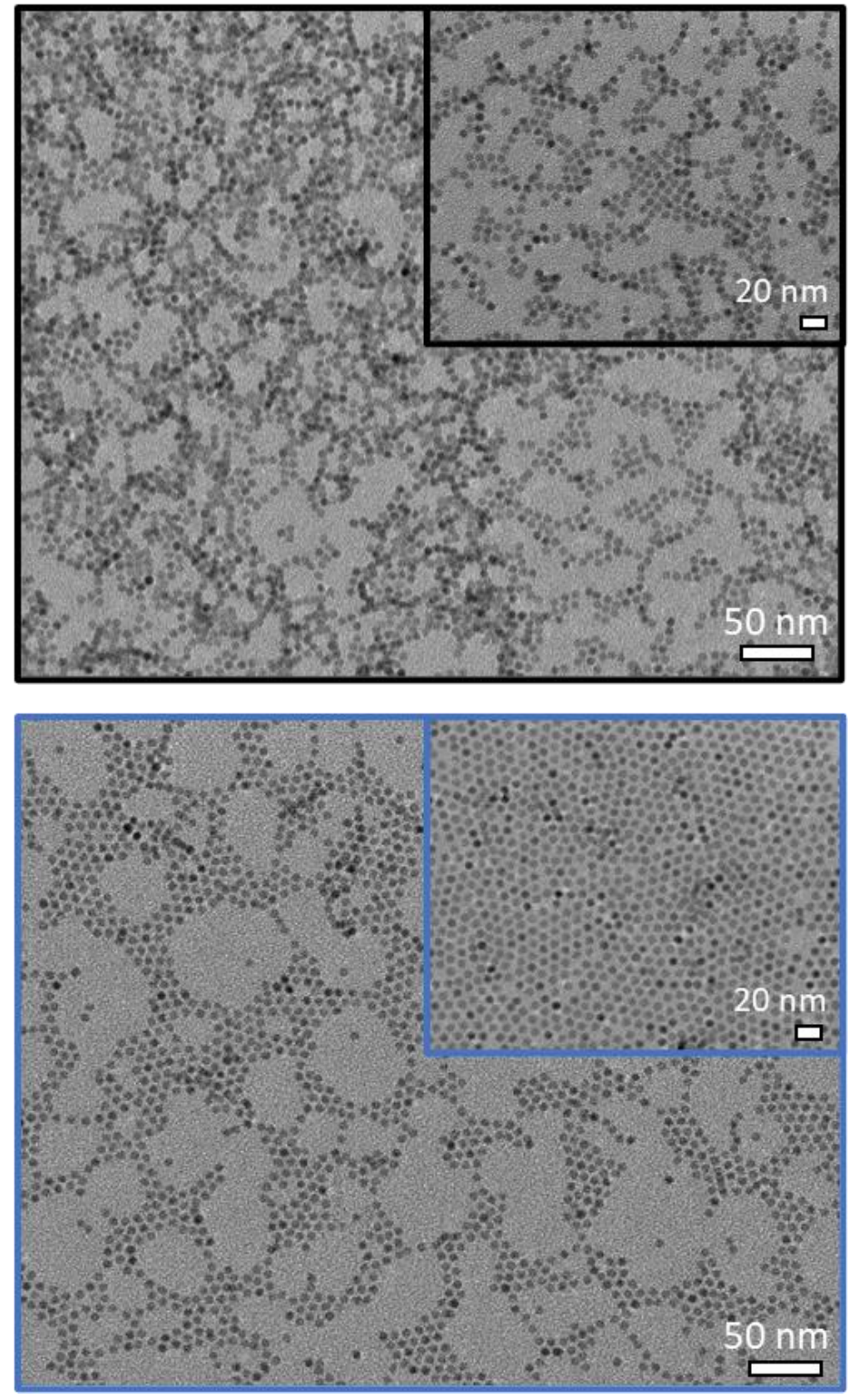

Figure 19: Top image represent a TEM micrograph of native PbS-eCl NCs purified through our acetone wash (See Experimental). These NCs display a random packing. After exposing the NCs to OLA+ OA and further purification, the same batch of NCs self-assembles into a superstructure displaying hexagonal close-packing. 


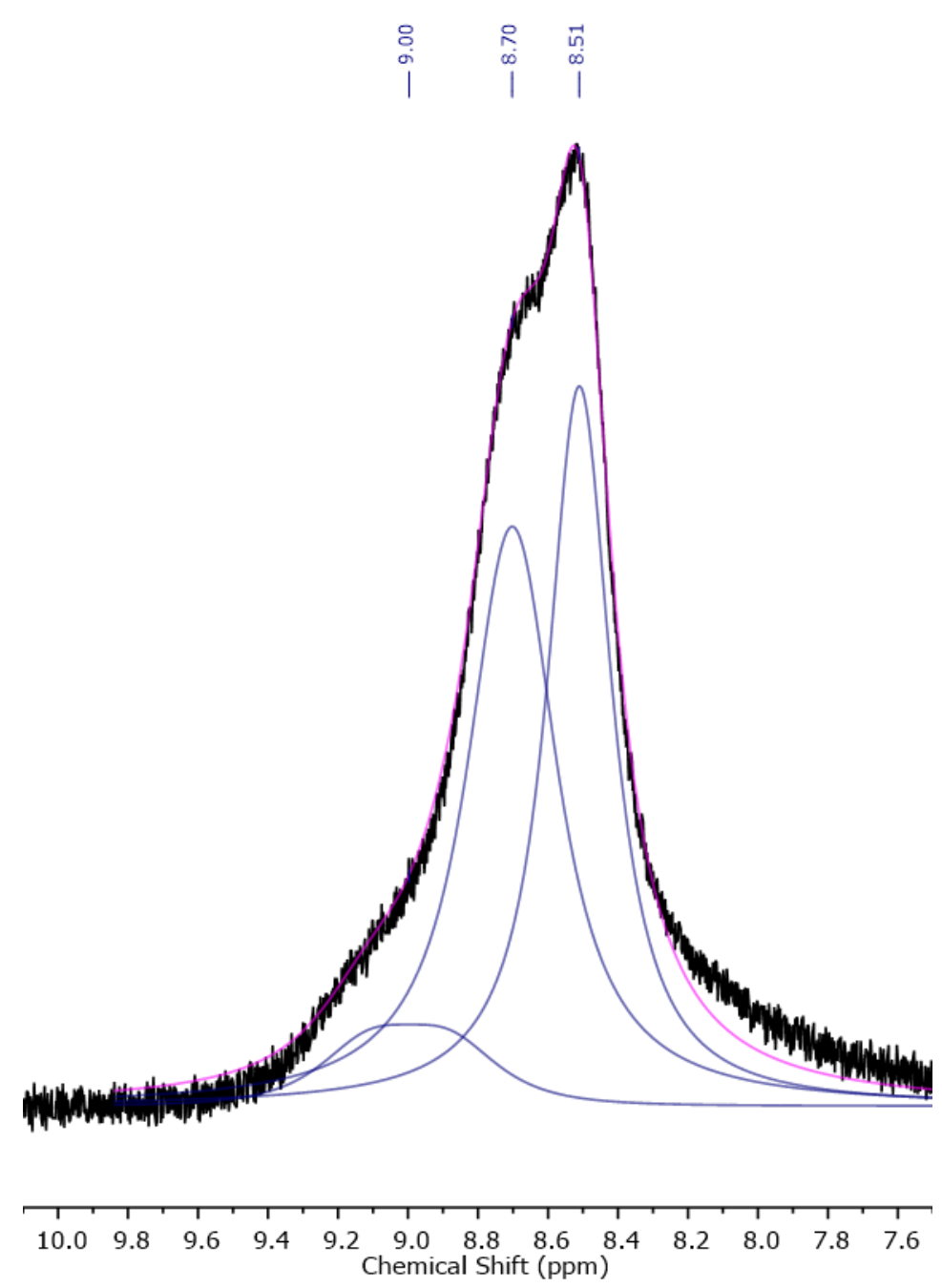

Figure S20: Fitted ammonium peak of a PbS-eCl ensemble exposed to OLA and UDA. This peak is well represented by three resonances, consistent with the presence of bound oleylammonium, oleylammonium-carboxylate and oleylammonium-chloride. 
Supplemental Section 4: 2D perovskites and other

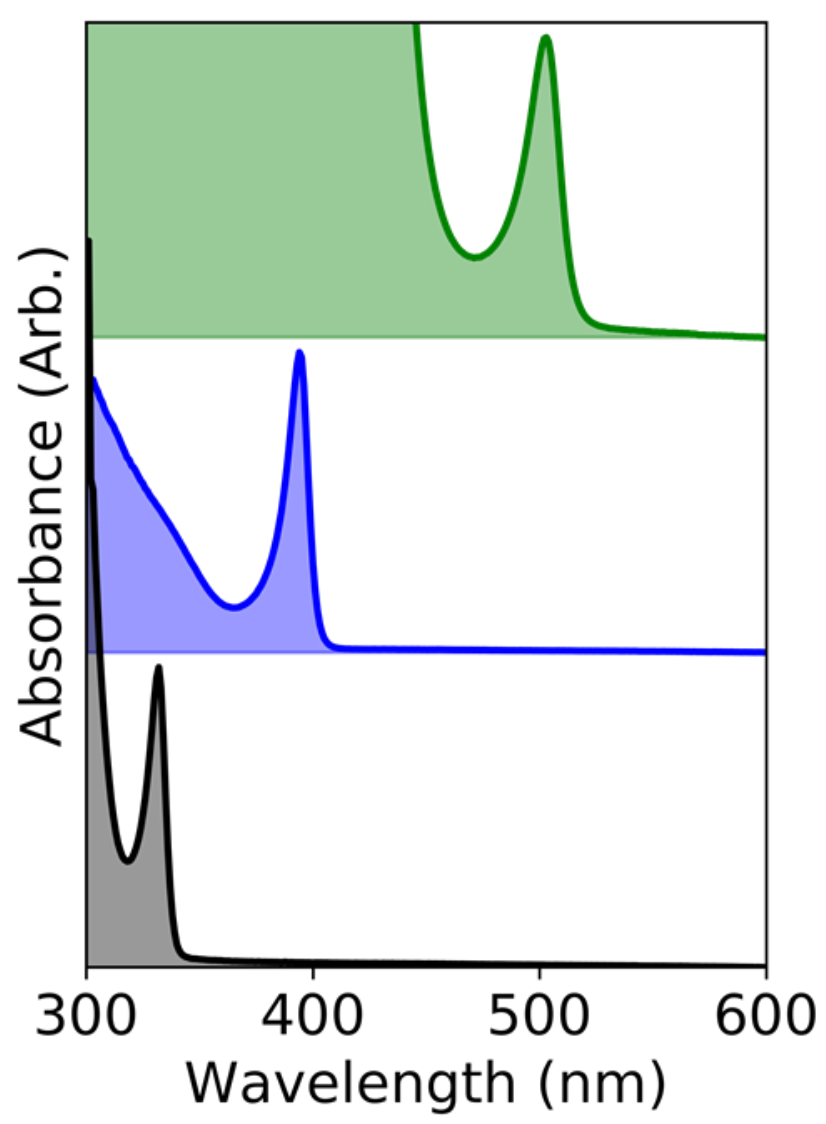

Figure S21: Optical absorption spectrum of 2D perovskite observed in the synthesis of PbS-eCl. Black: $\mathrm{L}_{2} \mathrm{PbCl}_{4}$, Blue: $\mathrm{L}_{2} \mathrm{PbBr}_{4}$, and Green: $\mathrm{L}_{2} \mathrm{Pbl}_{4}{ }^{2}$ 


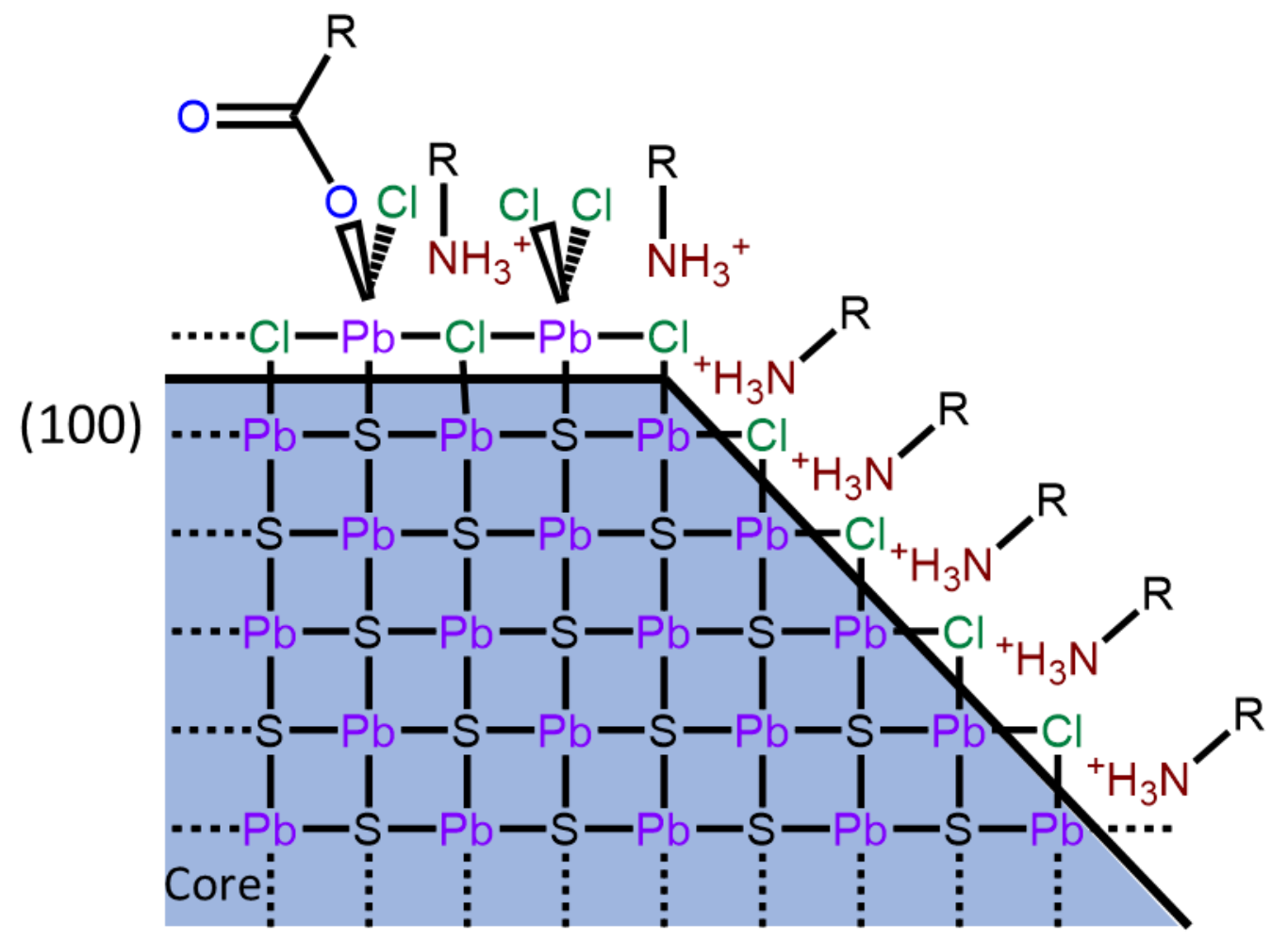

(111)

Figure S22: Schematic of an alternative surface structure to that proposed in Figure 5. 


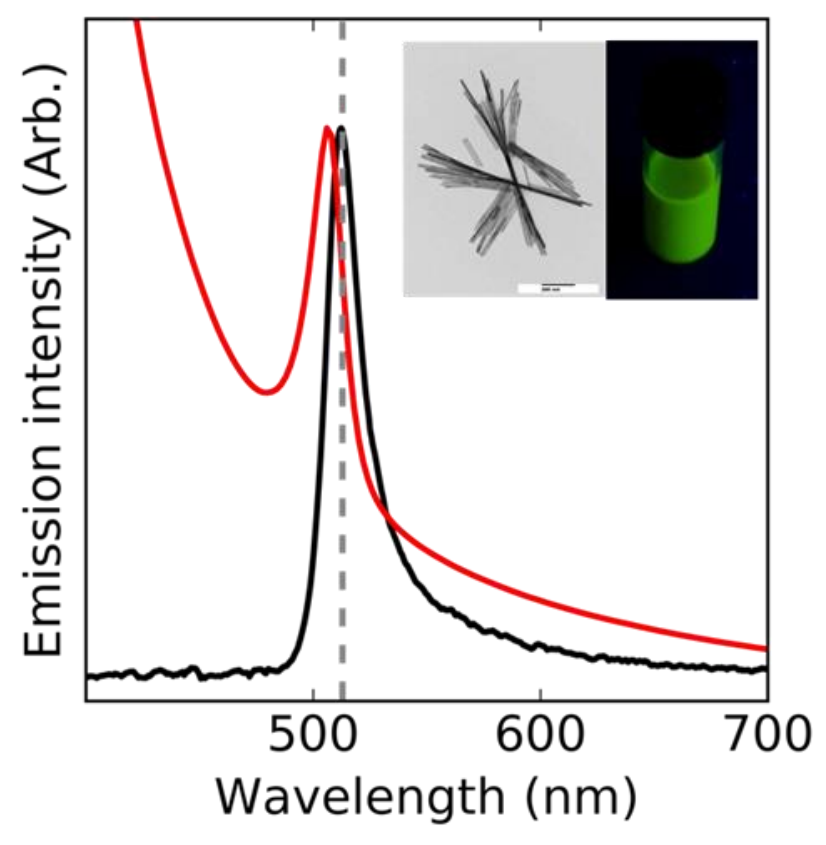

Figure S23: The precipitate obtained after the addition of antisolvent to PbS-el is a 2D perovskite. Shown is the optical absorption and emission that are characteristic of $\mathrm{L}_{2} \mathrm{Pbl}_{4}{ }^{2}$ Inset shows the platelet like structure of this material observed in TEM, and the bright green emission of the sample under UV light. 


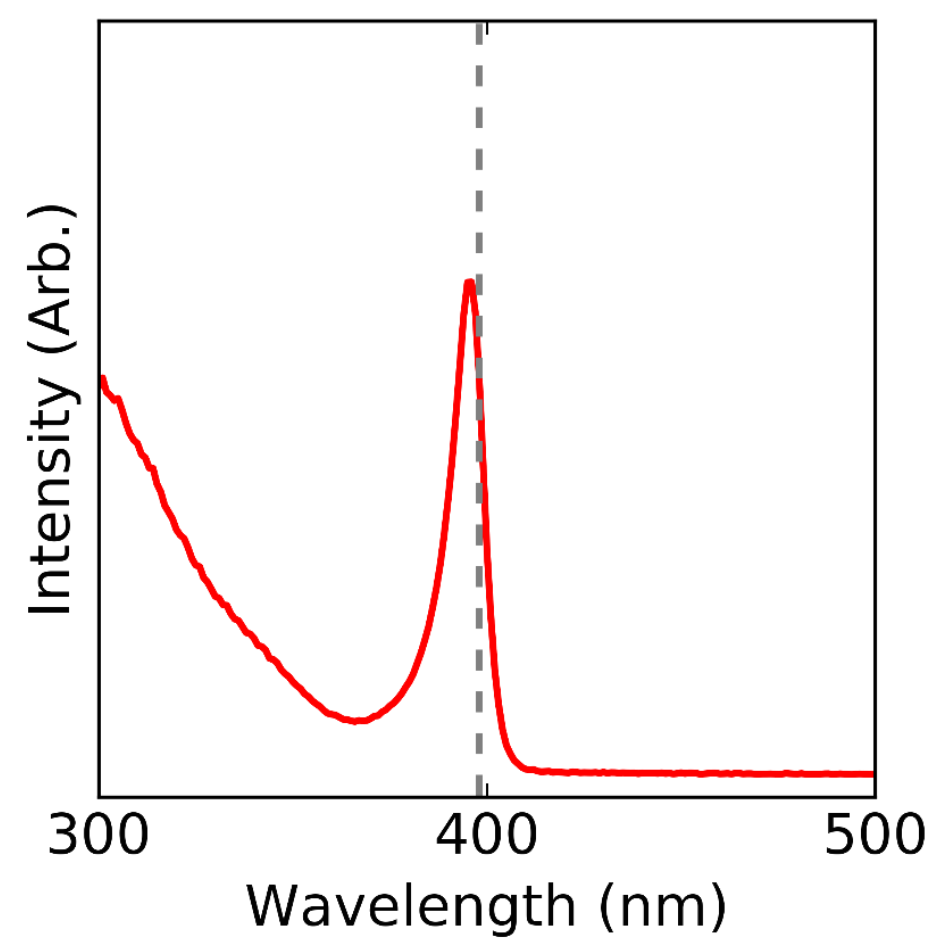

Figure S24: Precipitate obtained after the addition of antisolvent to $\mathrm{PbS}-\mathrm{eBr}$ is a 2D perovskite. Shown is the optical absorption and emission that are characteristic of $\mathrm{L}_{2} \mathrm{PbBr}_{4}{ }^{2}$ 


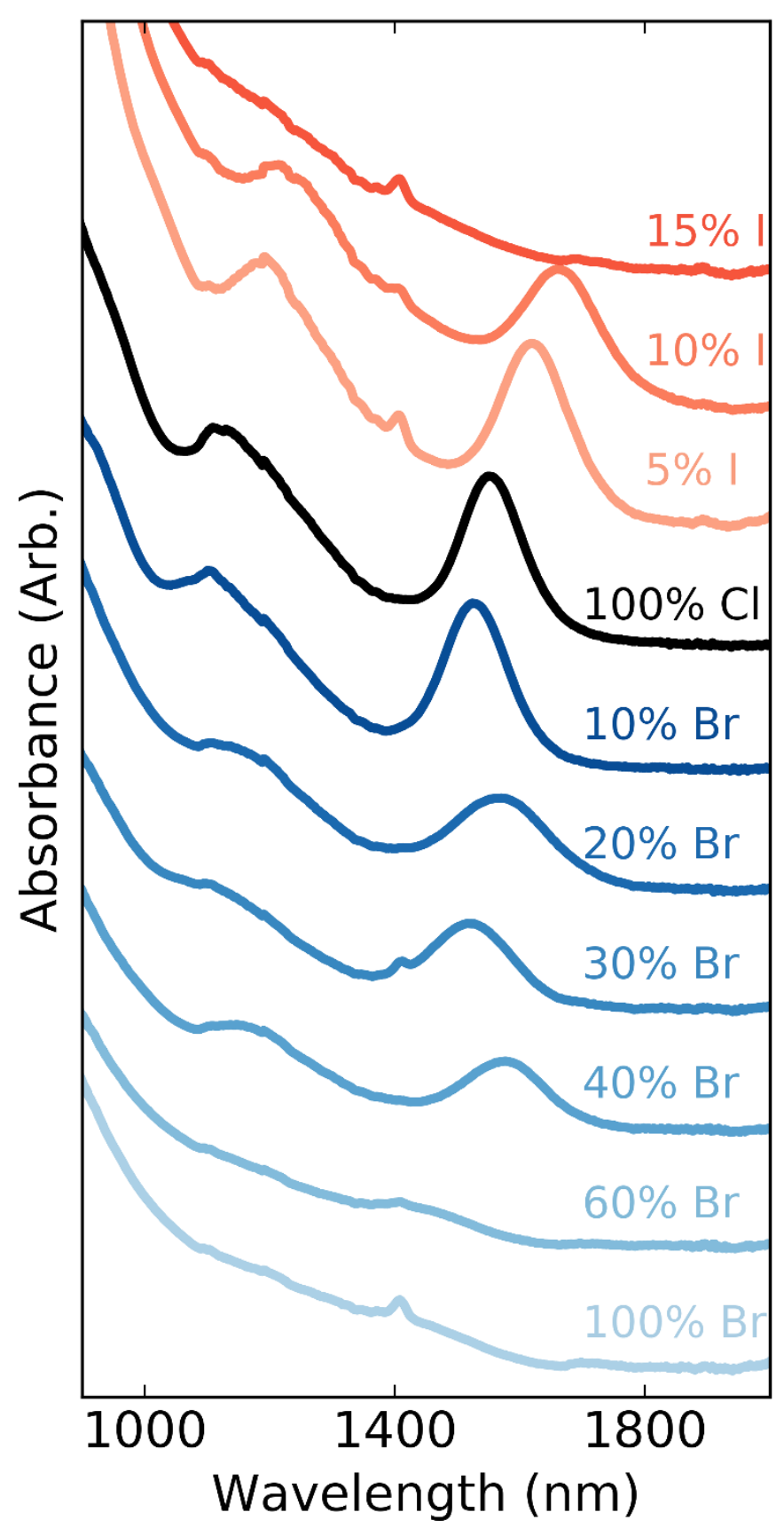

Figure S25: Optical absorption spectrum of PbS NCs produced with mixed lead halides. We observed that above a $15 \%$ content of $\mathrm{Pbl}_{2}$ the NCs loose their characteristic first excitonic peak and only a broad continuum is observed, indicative of a large size dispersity. In contrast up to $60 \% \mathrm{PbBr}_{2}$ content is required for the same effect. 

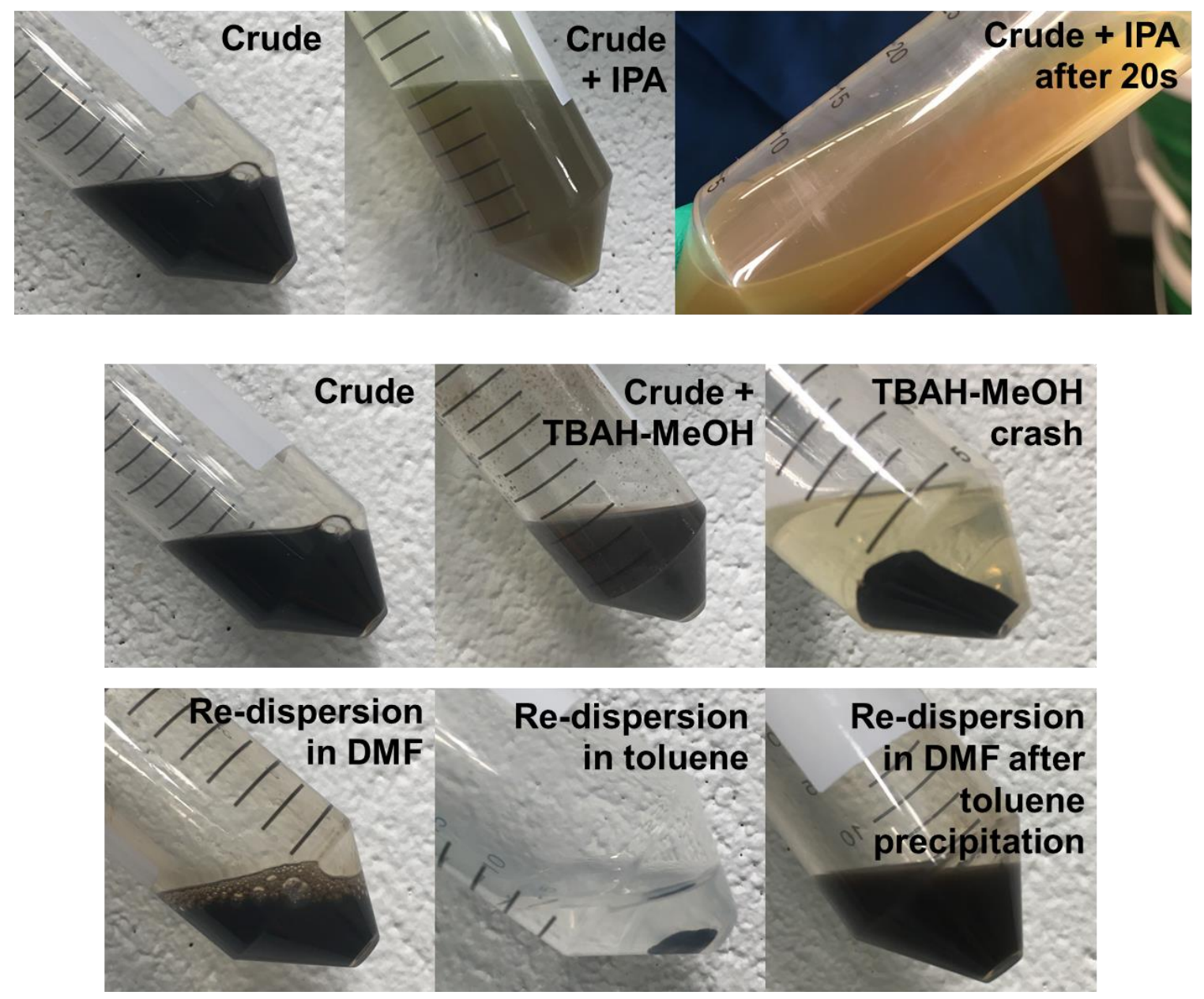

Figure S26: PbS-el are very sensitive to the addition of antisolvent. On the top we show a picture of an ensemble of PbS-el as obtained and after the addition of an antisolvent such as IPA. This solution rapidly turns from black to turbid yellow and no PbS NCs could be collected. The same step but using methanol with ammonium hydroxide allows for the precipitation of a black pellet. These NCs could then be redispersed in DMF and not toluene. Addition of toluene to PbS-el in DMF resulted in the precipitation of the NCs. These could then be redispersed again in DMF. 


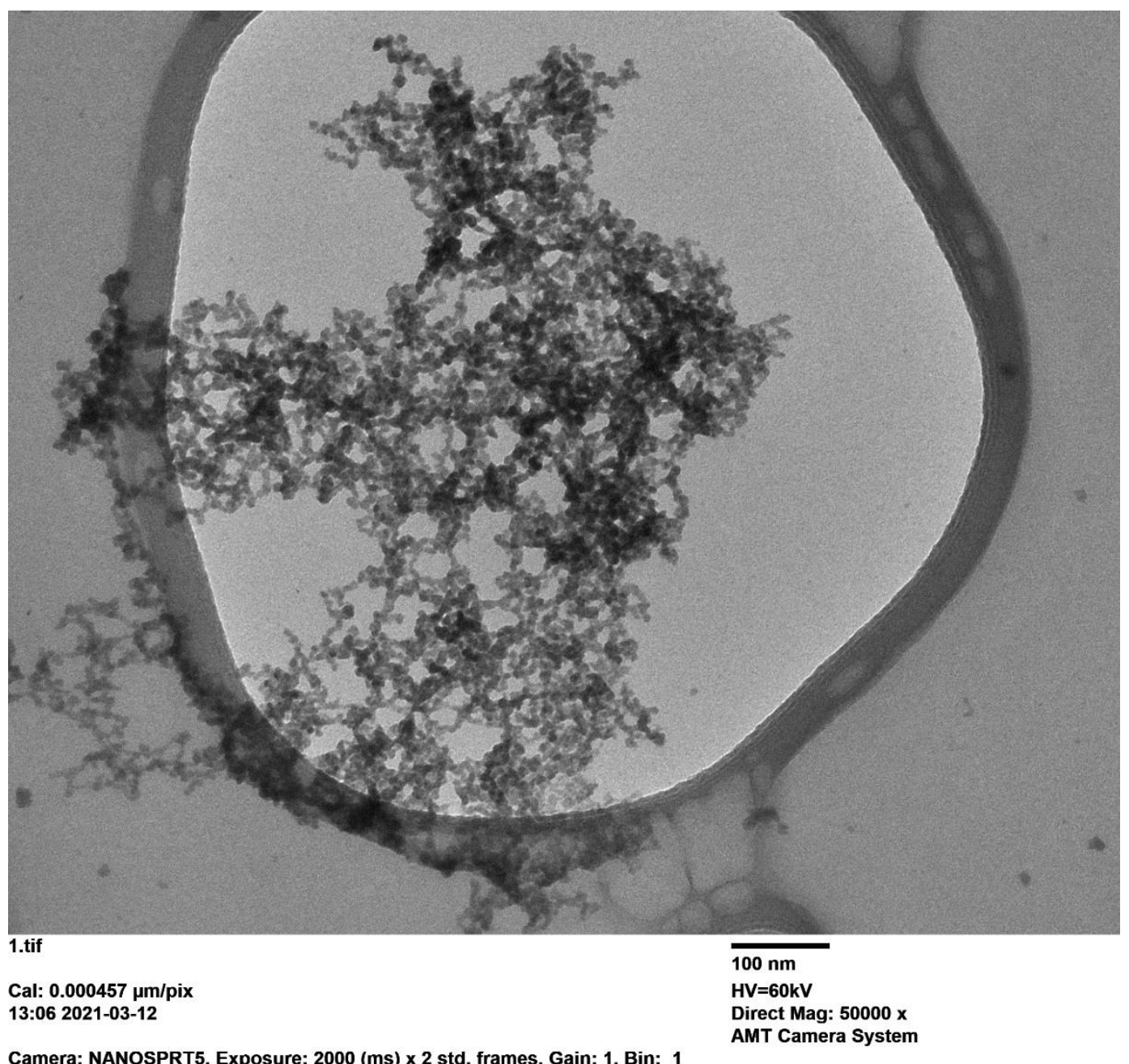

Camera: NANOSPRT5, Exposure: 2000 (ms) x 2 std. frames, Gain: 1, Bin: 1

Gamma: 1.00, No Sharpening, Normal Contrast

Figure S27: TEM micrograph of PbS NCs prepared directly from $\mathrm{Pbl}_{2}$ in DMF. These NCS formed large aggregates but remained colloidally stable in solution. 


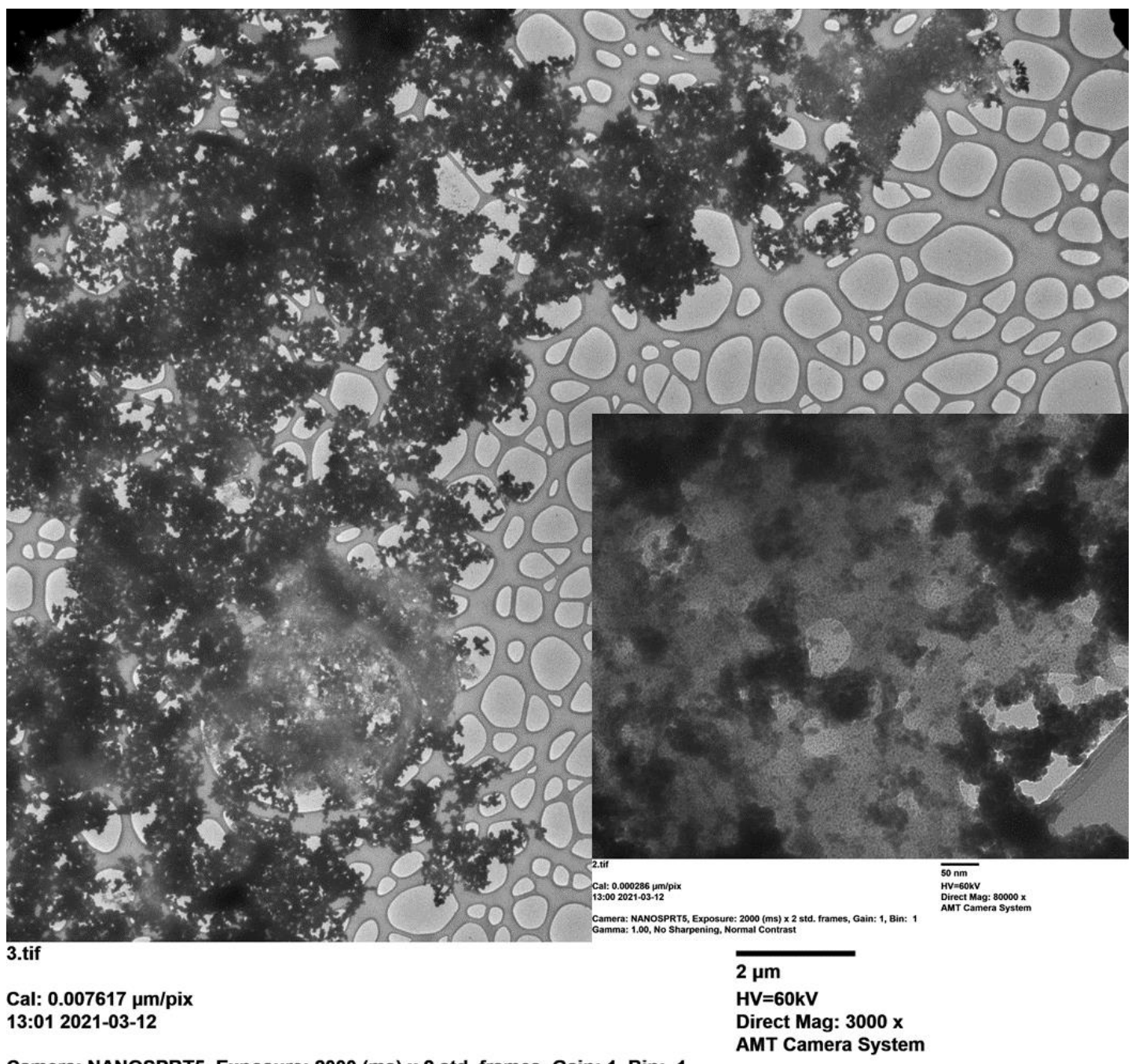

Camera: NANOSPRT5, Exposure: 2000 (ms) x 2 std. frames, Gain: 1, Bin: 1 Gamma: 1.00, No Sharpening, Normal Contrast

Figure S28: TEM micrograph of PbS-el purified with the addition of ammonium-hydroxide in methanol. The NCs form large aggregates, similar to PbS NCs from $\mathrm{Pbl}_{2}$ produced directly in DMF. 


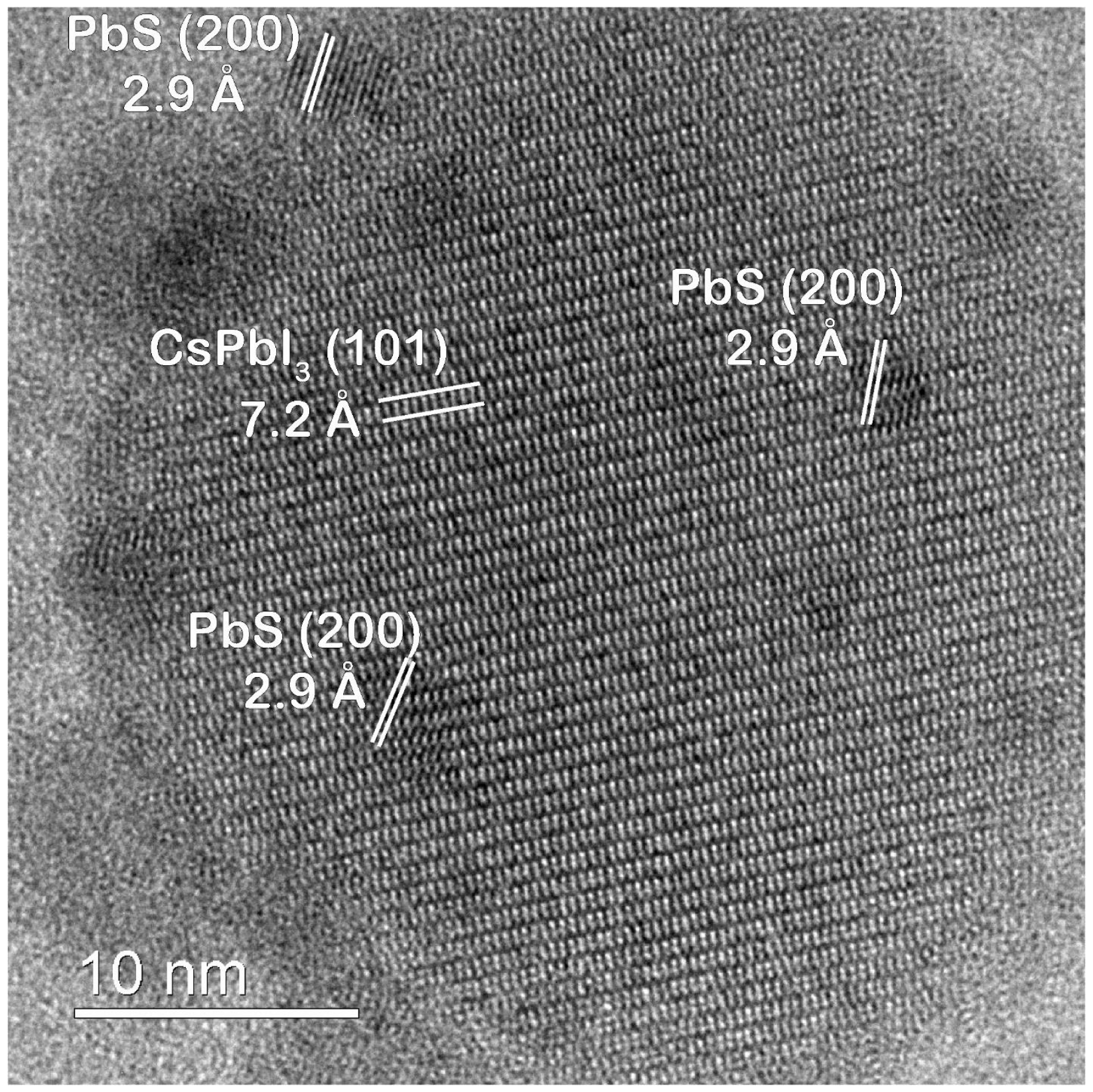

Figure S29: HR-TEM of PbS NCs in a $\mathrm{CsPbl}_{3}$ matrix. These particles were produced through the addition of Cs-oleate at room temperature to an unpurified reaction of PbS-el NCs. Two antisolvent purifications were then performed using ethyl acetate, as is common for the purification of $\mathrm{CsPbl}_{3} \mathrm{NCs}^{3}$ We obtained particles such as the one presented above. In this image. Two type of structures can be observed, and it appears that small NCs $\left(\varnothing^{\sim} 5 \mathrm{~nm}\right)$ are embedded into a larger particle $\left(\varnothing^{\sim} 30 \mathrm{~nm}\right)$. The observed lattice spacings are consistent with the smaller particles being $\mathrm{PbS}$ and the larger one $\mathrm{CsPb}_{3} .{ }^{4}$ Further measurements will be required to demonstrate that this particle is truly a heterostructure with epitaxially matched $\mathrm{PbS}$ and $\mathrm{CsPbl}_{3}$. 


\section{References}

(1) Weidman, M. C.; Beck, M. E.; Hoffman, R. S.; Prins, F.; Tisdale, W. A. Monodispers, Air Stable PbS Nanocrystals via Precursor Stoichiometry Control. ACS Nano 2014, No. 6, 6363-6371.

(2) Weidman, M. C.; Seitz, M.; Stranks, S. D.; Tisdale, W. A. Highly Tunable Colloidal Perovskite Nanoplatelets through Variable Cation, Metal, and Halide Composition. ACS Nano 2016, 10 (8), 7830-7839.

(3) Swarnkar, A.; Marshall, A. R.; Sanehira, E. M.; Chernomordik, B. D.; Moore, D. T.; Christians, J. A.; Chakrabarti, T.; Luther. Quantum Dot-Induced Phase Stabilization of a-CsPbl3 Perovskite for HighEffiency Photovoltaics. Science 2016, 354 (6308), 92-96.

(4) Sutton, R. J.; Filip, M. R.; Haghighirad, A. A.; Sakai, N.; Wenger, B.; Giustino, F.; Snaith, H. J. Cubic or Orthorhombic? Revealing the Crystal Structure of Metastable Black-Phase CsPbl3 by Theory and Experiment. ACS Energy Lett. 2018, 3 (8), 1787-1794. 